\title{
Whole-limb scaling of muscle mass and force-generating capacity in amniotes
}

\author{
Peter J Bishop $^{\text {Corresp., } 1,2 \text {, Mark A Wright }}{ }^{2}$, Stephanie E Pierce ${ }^{2}$ \\ ${ }^{1}$ Geosciences Program, Queensland Museum, Brisbane, Queensland, Australia \\ 2 Department of Organismic and Evolutionary Biology, Harvard University, Cambridge, Massachusetts, United States of America \\ Corresponding Author: Peter J Bishop \\ Email address: pbishop@fas.harvard.edu
}

Skeletal muscle mass, architecture and force-generating capacity are well known to scale with body size in animals, both throughout ontogeny and across species. Investigations of limb muscle scaling in terrestrial amniotes typically focus on individual muscles within select clades, but here this question was examined at the level of the whole limb across amniotes generally. In particular, the present study explored how muscle mass, forcegenerating capacity (measured by physiological cross-sectional area) and internal architecture (fascicle length) scales in the fore- and hindlimbs of extant mammals, nonavian saurians ('reptiles') and bipeds (birds and humans). Sixty species spanning almost five orders of magnitude in body mass were investigated, comprising previously published architectural data and new data obtained via dissections of the opossum Didelphis virginiana and the tegu lizard Salvator merianae. Phylogenetic generalized least squares was used to determine allometric scaling slopes (exponents) and intercepts, to assess whether patterns previously reported for individual muscles or functional groups were retained at the level of the whole limb, and to test whether mammals, reptiles and bipeds followed different allometric trajectories. In general, patterns of scaling observed in individual muscles were also observed in the whole limb. Reptiles generally have proportionately lower muscle mass and force-generating capacity compared to mammals, especially at larger body size, and bipeds exhibit strong to extreme positive allometry in the distal hindlimb. Remarkably, when muscle mass was accounted for in analyses of muscle force-generating capacity, reptiles, mammals and bipeds almost ubiquitously followed a single common scaling pattern, implying that differences in whole-limb forcegenerating capacity are principally driven by differences in muscle mass, not internal architecture. In addition to providing a novel perspective on skeletal muscle allometry in animals, the new dataset assembled was used to generate pan-amniote statistical relationships that can be used to predict muscle mass or force-generating capacity in extinct amniotes, helping to inform future reconstructions of musculoskeletal function in 
the fossil record. 


\section{Whole-limb scaling of muscle mass and force-generating \\ 2 capacity in amniotes}

3

4

5 Peter J. Bishop ${ }^{1,2, *}$, Mark A. Wright ${ }^{1}$ and Stephanie E. Pierce ${ }^{1}$

6

7

$8{ }^{1}$ Museum of Comparative Zoology and Department of Organismic and Evolutionary Biology,

9 Harvard University, Cambridge, Massachusetts, USA

$10{ }^{2}$ Geosciences Program, Queensland Museum, Brisbane, Queensland, Australia

11

12

13

14

15

16

17

18

19

20

21

22

23

24

25

26

27

28

29

30

*Correspondence to: pbishop@,fas.harvard.edu

4

5

16

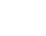


31 Abstract

32

33

Skeletal muscle mass, architecture and force-generating capacity are well known to scale with body size in animals, both throughout ontogeny and across species. Investigations of limb muscle scaling in terrestrial amniotes typically focus on individual muscles within select clades, but here this question was examined at the level of the whole limb across amniotes generally. In particular, the present study explored how muscle mass, force-generating capacity (measured by physiological cross-sectional area) and internal architecture (fascicle length) scales in the foreand hindlimbs of extant mammals, non-avian saurians ('reptiles') and bipeds (birds and humans). Sixty species spanning almost five orders of magnitude in body mass were investigated, comprising previously published architectural data and new data obtained via dissections of the opossum Didelphis virginiana and the tegu lizard Salvator merianae. Phylogenetic generalized least squares was used to determine allometric scaling slopes (exponents) and intercepts, to assess whether patterns previously reported for individual muscles or functional groups were retained at the level of the whole limb, and to test whether mammals, reptiles and bipeds followed different allometric trajectories. In general, patterns of scaling observed in individual muscles were also observed in the whole limb. Reptiles generally have proportionately lower muscle mass and force-generating capacity compared to mammals, especially at larger body size, and bipeds exhibit strong to extreme positive allometry in the distal hindlimb. Remarkably, when muscle mass was accounted for in analyses of muscle force-generating capacity, reptiles, mammals and bipeds almost ubiquitously followed a single common scaling pattern, implying that differences in whole-limb force-generating capacity are principally driven by differences in muscle mass, not internal architecture. In addition to providing a novel perspective on skeletal muscle allometry in animals, the new dataset assembled was used to generate pan-amniote statistical relationships that can be used to predict muscle mass or force-generating capacity in extinct amniotes, helping to inform future reconstructions of musculoskeletal function in the fossil record. 


\section{$62 \underline{\text { Introduction }}$}

63

64 Countless aspects of organismal biology vary with the size of the organism as a whole (Schmidt-

Nielsen 1985; Vogel 2003). In a mechanical context, the differential scaling of lengths, areas, volumes, inertias and other metrics with increasing size (e.g., body mass) influences an organism's ability to generate and resist forces. An oft-cited example is the comparison between the cross-sectional area of a tissue and the gravitational loads it must bear. Under isometric (geometric) scaling, area scales proportional to length ${ }^{2}$, or assuming a constant tissue density, $\operatorname{mass}^{2 / 3}$; in contrast, gravity-induced loads scale proportional to mass ${ }^{1}$. Thus, larger species would have disproportionately less tissue area to support their mass, incurring greater tissue stress (= force per unit area). Given that tissue mechanical properties are largely constant across species (e.g., yield strength of cortical bone; Currey 2002), this scaling relationship is undesirable, and usually necessitates changes to organismal structure, behaviour or performance to mitigate the effects of increasing body size, such as larger species using a more extended limb posture during stance and gait (Biewener 1989; Dick \& Clemente 2017).

The macroscopic architecture of skeletal muscle is one such aspect of organismal structure that is known to scale with body size in animals. A particularly relevant architectural metric is its physiological cross-sectional area (PCSA), which relates a muscle's mass with the length and arrangement of its constituent fascicles (Calow \& Alexander 1973; Gans \& Bock 1965; Sacks \& Roy 1982), and can be used to estimate the muscle's maximal force output in an isometric contraction (Powell et al. 1984; Medler 2002). If animals were isometrically scaled, muscle mass would scale in direct proportion to total body mass, fibre length would scale proportional to $\operatorname{mass}^{1 / 3}$, and PCSA - and therefore force-producing capacity - would scale proportional to $\operatorname{mass}^{2 / 3}$. Larger species would therefore be relatively weaker. Over the past five decades, numerous studies have examined the interspecific scaling of muscle mass and force-generating capacity (via PCSA) across a wide variety of species, in mammals (Alexander 1977; Alexander et al. 1981; Bennett \& Taylor 1995; Cuff et al. 2016a; Cuff et al. 2016b; Eng et al. 2008; McGowan et al. 2008; Pollock \& Shadwick 1994), birds (Bennett 1996; Maloiy et al. 1979) and non-avian saurians (Allen et al. 2015; Cieri et al. 2020; Dick \& Clemente 2016). Although the caveat must be acknowledged that different studies have used different line-fitting approaches to 
93

94

95

96

97

98

99

100

101

102

103

104

105

106

107

108

109

110

111

112

113

114

115

116

117

118

119

120

121

122

estimate scaling exponents, these studies have shown that across most species the architecture of individual muscles scales in a broadly similar fashion. Muscle mass typically exhibits weak negative to modest positive allometry (range of exponents $0.9-1.15$ ), PCSA typically exhibits weak to modest positive allometry (range of exponents $0.69-0.91$ ), and fascicle length typically exhibits modest positive to modest negative allometry (range of exponents $0.14-0.5$ ). Some notable exceptions to this include the extreme positive allometry in the hindlimb extensors of macropod marsupials (mass exponents 1.11-1.61, PCSA exponents 0.78-1.33; Bennett \& Taylor 1995; McGowan et al. 2008) and many forelimb muscles in varanid lizards (mass exponents 0.95-1.48, PCSA exponents 0.75-1.15; Cieri et al. 2020), and near mass independence of fascicle length in the plantaris of mammals (Pollock \& Shadwick 1994; although this may be affected by sampling in that particular study). The common occurrence of positive allometry helps reduce the 'strength deficit' experienced by larger animals, but this deficit is fully counteracted only in extreme cases of positive allometry, where the PCSA exponent exceeds 1.

It remains unknown as to how the aforementioned scaling patterns for individual muscles translate to scaling at the level of the whole limb. Likewise, it remains unknown as to whether architectural disparities observed between muscles, functional groups of muscles (e.g., ankle extensors) or even species remain at the level of the whole limb. The limbs of an animal must exist and function as a single integrated entity, which imposes at least two requirements on the constituent muscles:

1. The muscles collectively share a common volume, whereby changes in the size of one muscle may affect the size of adjacent muscles (Fig. 1A). For example, in order for the limb to avoid becoming too heavy (or having too high a rotational inertia), increase in the size of one muscle may necessitate a decrease in the size of another.

2. Architectural specialization of muscles can limit their ability to effectively contribute to a diverse range of tasks (Wilson \& Lichtwark 2011), and hence specialization in one muscle may necessitate concomitant change in another so that limb functionality is not compromised. For example, a muscle with short fascicles will have a high PCSA but a reduced working range, which may require another muscle to compensate by having longer fascicles, but with reduced PCSA (Fig. 1B). 
123 These two requirements imply that animal limbs are subject to a 'fascicle packing problem', and

124 raise the question of how flexible (evolutionarily labile) animal limbs are in terms of their

125 muscular anatomy. Are there different strategies for packing PCSA into a given volume of limb

126 muscle, or do the differences observed between individual muscles and between species 'cancel

127 out' at the level of the whole limb? Depending on how stringent the above requirements are, the

128 total force-producing capacity (strength) of an animal's limb may therefore be strongly tied to

129 total limb muscle mass, irrespective of a given species' size or functional requirements.

Understanding how whole-limb muscle mass and force-generating capacity relate to one another,

132

133

134

135

136

137

138

139

140

141

142

143

144

145

146

147

148

149

150

151

152

153 and how this relation scales with body size, is not just pertinent to the study of extant species. Muscle force-generating capacity is a key unknown in studies of extinct animal function and behaviour, and empirical data from extant species play a vital role in informing inferences of extinct species (Bates \& Falkingham 2018; Bishop et al. 2021a; Fahn-Lai et al. 2020; Lautenschlager et al. 2018; Sellers et al. 2013). The use of empirical data from extant members of a specific clade may be appropriate for extinct members of that clade (e.g., using data derived from extant palaeognath birds to guide inferences of extinct palaeognath birds; Bishop 2015), but it is not immediately clear how this should be approached for extinct species that are phylogenetically distant or morphologically disparate from extant species. In particular, the more phylogenetically distant or morphologically disparate an extinct taxon is, the lower the confidence that can generally be placed in inferences of muscle origin or insertion (Carrano \& Hutchinson 2002; Witmer 1995), relative size, internal architecture and even whether a given muscle exists in the extinct taxon (e.g., differentiation from adjacent muscles). Developing an understanding of how muscle mass and force-generating capacity scales with body size across a wide range of species, even at a broad anatomical resolution such as the whole limb, could therefore provide a useful starting point for better informed inferences of extinct taxa. Even if just overall bulk and strength of limb musculature were able to be confidently constrained, this would represent a practical advance upon which future refinements could be made.

The present study sought to address the above outstanding issues by conducting a holistic assessment of whole-limb muscle mass and force-generating capacity in extant terrestrial amniotes. It had three key aims: (1) to contribute new data from hitherto unsampled clades 
154 (ameridelphian marsupials and lacertoid lizards), broadening the diversity of the available 155 comparative dataset; (2) to investigate how muscles scale with body size at the level of the whole 156 limb, in both the fore- and hindlimb, providing a first assessment of the stringency of the

157 'fascicle packing problem'; (3) to derive generic, amniote-wide statistical predictive

158

159

160

161

162

163

164

165

166

167

168

169

170

171

172

173

174

175

176

177

178

179

180

181

182

183

184 relationships that would have utility in deriving inferences of musculature in extinct amniote species. This study is the first comparative synthesis of muscle architecture scaling across extant mammals, birds and non-avian saurians. In addition to providing a novel perspective on the topic of muscle scaling in animals, the results from this study provides a platform for more rigorous inference of muscle strength in extinct amniote clades.

\section{Materials \& Methods}

\section{Dataset}

The present study is founded upon muscle architecture data derived from dissections (Table 1); all raw data used are provided in Table S1.The sources of the data are described in the following two subsections. Although this study addresses 'whole limb' scaling, it is restricted in scope to the musculature crossing the primary three joints of the limbs (shoulder/hip, elbow/knee and wrist/ankle), ignoring the intrinsic musculature of the manus and pes. This was necessitated by the practical difficulties of accurately dissecting and measuring the latter muscles (particularly for small species), such that data are very rarely reported in the comparative literature. Ignoring manual and pedal muscles is also justified given that they comprise a small fraction of total limb musculature, and presumably contribute only a small fraction towards limb support and propulsion during locomotor activities. Although extrinsic shoulder muscles attaching to the scapula can be important for locomotion in therian mammals (Hudson et al. 2011a; Jenkins \& Weijs 1979), these were excluded from consideration to facilitate a fair comparison across all species; only muscles that explicitly attached to the humerus or more distal forelimb skeleton were included. The forelimb dataset comprised architectural measurements for a total of 912 muscles in 31 species (21 mammals and 10 non-avian saurians, the latter hereafter referred to as 'reptiles' for simplicity) spanning more than four orders of magnitude in body mass, and the 
185 hindlimb dataset comprised measurements for a total of 1,181 muscles in 36 species (19

186

187

188

189

190

191

192

193

194

195

196

197

198

199

200

201

202

203

204

205

206

207

208

209

210

211

212

213

mammals, 12 reptiles and 5 birds) spanning almost five orders of magnitude in body mass. In the hindlimb dataset, birds and humans were collectively treated as a single group, 'bipeds', such that 'mammals' herein refer to all mammals except humans. Given the small sample size (and taxonomic skew) of birds in the current dataset, and the broad aims of the study, it was not considered justifiable to analyze birds and humans as separate entities here. Furthermore, as the present study concerns terrestrial amniotes, the forelimb of bipeds was not investigated.

For each muscle, its physiological cross-sectional area (PCSA) was calculated as

$$
\operatorname{PCSA}=\frac{m_{\text {muscle }} \cdot \cos \left(\alpha_{\mathrm{O}}\right)}{\rho \cdot \mathrm{I}_{\mathrm{O}}},
$$

where $m_{\text {muscle }}$ is belly mass, $\alpha_{\mathrm{o}}$ is pennation angle, $\ell_{\mathrm{o}}$ is fascicle (or 'fibre') length and $\rho$ is muscle tissue density, the latter taken to be constant for vertebrate skeletal muscle at $1,060 \mathrm{~kg} / \mathrm{m}^{3}$

(Hutchinson et al. 2015; Mendez \& Keys 1960). It is important to note that this equation assumes that all fascicles of a muscle act in parallel in generating force, allowing their individual crosssectional areas to be summed (see also Sacks \& Roy 1982). In reality, the constituent fibres of a given fascicle are often shorter than the fascicle itself, wherein their ends overlap or interdigitate (Gaunt \& Gans 1992; Infantolino et al. 2012), although they may still activate simultaneously with one another, thus functioning as a single fibre (Bodine et al. 1982). Additionally, as per common practice, it was assumed that measurements of fascicle length and pennation angle corresponded to the muscle's optimal fibre length and optimal pennation angle, respectively (Zajac 1989).

\section{$\underline{\text { Published data }}$}

The majority of data used in this study were sourced from previously published comparative studies on muscle architecture, biomechanics or scaling (see Table 1 for references; see also Martin et al. 2020 for a review of methods used to measure muscle architecture). Unfortunately, many of the earlier studies on muscle architecture scaling cited in the Introduction did not report 
214 their raw measurements and so their data were unable to be incorporated into a taxonomically

215 richer analysis. Literature data were selected according to three requirements:

216 1. All or almost all of the muscles of the limb had been measured and reported (save the

217 manual and pedal muscles, as noted above), since the overarching aim of the present study is

218 considering the whole limb. Studies in which a few small muscles (e.g., deep muscles such

219 as the gemellus, quadratus femoris or popliteus) were not reported were still included, since the omission of such small muscles is expected to have minimal effect on the overall results. Studies that measured multiple muscles under a single name (e.g., multiple heads of the flexor carpi radialis as a single muscle) were also included, since this nevertheless accounts for all the muscle mass present. In contrast, studies that did not report one or more major muscles were excluded from consideration. All datasets ultimately selected for use in the present study included all major extensor and adductor (antigravity) muscles of the limb. The specific muscles included (and which, if any, were excluded) in a given source study are outlined in Table S1.

2. Each of the architectural parameters listed in Equation 1 were either explicitly reported, or their values able to be back-calculated from reported values, the latter either as PCSA (e.g., Cuff et al. 2016a,b; back-calculated via Equation 1) or mass-scaled values (e.g., Dick and Clemente 2017, Cieri et al. 2020; back-calculated by unscaling according to reported body mass). A few previous studies had neglected to measure (or at least report) pennation angle, but to maximize consistency across the present dataset these studies were excluded. Studies that reported dry muscle mass only were also excluded.

3. The data reported were for adult or large-sized individuals, to reduce potential confounding effects of ontogenetic variation (Table S1). In approximately two-fifths of species sampled, multiple similarly-sized individuals had been investigated, but the data reported by the relevant studies were only presented as a species mean, wherein a given architectural parameter for a given muscle had already been averaged across the individuals studied; in these cases, the mean body mass of that sample was used. For all other species, architectural and body mass data were reported for separate individuals. When data for multiple individuals of a given species had been reported separately (e.g., Lamas et al. 2014, Allen et al. 2015, Martin et al. 2019), those for the largest individual were used, to reduce possible ontogenetic effects. This approach was deemed more appropriate than computing a species 
245

246

247

248

249

250

251

252

253

254

255

256

257

258

259

260

261

262

263

264

265

266

267

268

269

270

271

272

273

274

275

average across all individuals, because in several instances the sample of individuals investigated by prior studies (especially those focused on ontogeny) exhibited high disparity in body sizes, undermining the value of a species mean; moreover, such an arithmetic mean would not account for ontogenetic allometry within the species, and hence could introduce further error into the analyses. Ultimately, each species contributed only a single datapoint to the analyses.

Data were also sourced for a further 15 species from six additional studies, which had reported just muscle mass (Table $\mathrm{S} 1$ ). These studies either reported mass of each individual muscle separately (Hudson et al. 2011a; Hudson et al. 2011b; Ogihara et al. 2009; Payne et al. 2006; Zihlman et al. 2011), or reported total muscle mass for the limb as a whole (Grand 1977), and helped further increase taxonomic coverage in the final dataset.

New data

To broaden the taxonomic diversity of the dataset, and contribute novel data that future studies may draw upon, dissections were undertaken on a single adult individual each of the Virginia opossum (Didelphis viginiana) and the Argentine black and white tegu (Salvator merianae). The former is an ameridelphian marsupial, and apart from a single species of bandicoot (australidelphian; Martin et al., 2019) is the only other marsupial in the dataset; the latter is a lacertoid lizard, representing a hitherto unsampled part of squamate phylogeny. Intact whole carcasses of wild individuals were obtained as part of a prior study (Fahn-Lai et al. 2020), sourced from Worcester County, Massachusetts (D. virginiana, Massachusetts Division of Fisheries and Wildlife) and Everglades National Park, Florida (S. merianae, Daniel Beard Center, United States Geological Survey). Upon acquisition, the specimens were immediately frozen at $-20^{\circ} \mathrm{C}$, and subsequently fully thawed prior to dissection and architectural measurement. The right fore- and hindlimbs were dissected in both cases. Muscle architecture measurement followed standard dissection procedures (Allen et al. 2015; Biewener \& Full 1992; Fahn-Lai et al. 2020), using a magnifying lamp where necessary. To minimize desiccation of the fresh specimens, tissues were kept moistened with paper towel soaked in saline solution throughout. Muscle belly mass was measured with an electronic balance (Precisa 320 XB, 
276 Precisa Gravimetrics AG, Dietikon; $0.0001 \mathrm{~g}$ precision), fascicle length with digital calipers

277 (0.01 mm precision) and pennation angle with a transparent protractor ( $1^{\circ}$ precision).

278 Measurements of fascicle lengths and pennation angles were made at random locations

279 throughout a muscle belly, and depending on muscle size up to ten measures each were made,

280 after which the arithmetic mean was derived.

281

282

283

284

285

286

287

288

289

290

291

292

293

294

295

296

297

298

299

300

301

302

303

304

305

306

\section{Anatomical comparisons}

Four main, and two subsidiary, comparisons were undertaken for both fore- and hindlimbs, as described below. For each comparison, patterns were examined for the limb as a whole, as well as just the proximal and distal limb muscles separately, given that some previous studies have noted scaling differences between the proximal and distal limb for individual muscles or functional groups (Alexander et al. 1981; Cuff et al. 2016a; Cuff et al. 2016b; Dick \& Clemente 2016; Eng et al. 2008). 'Proximal' muscles were classified as those in which the majority of their bulk resides proximal to the elbow (forelimb) or knee (hindlimb) joints, whereas 'distal' muscles have the majority of their bulk residing distal to those joints. This volume-based approach is more relevant to the 'fascicle packing problem', and avoids the complications caused by flexor or extensor muscles crossing the joints involved. Furthermore, given that a substantial component of hindlimb locomotor muscle in reptiles is the caudofemoralis longus (CFL), which chiefly resides in the proximal tail, whole hindlimb and proximal hindlimb analyses were also rerun with this muscle excluded from the reptile sample. In addition to providing more nuanced insight into questions of scaling and fascicle packing, these additional variations provide greater sophistication to predictive relationships derived from the data. Ultimately, eight different subanalyses were performed for each anatomical comparison (48 in total).

\section{$\# 1$ - Total muscle mass $\left(\Sigma m_{\text {muscle }}\right) \mathrm{v}$. body mass $\left(m_{\text {body }}\right)$}

This comparison examines how much of total biomass is invested in limb musculature; under isometry, the scaling exponent would be 1. It ignores the potential for systematic variation in relative body composition in terms of other tissue types (bone, integument, etc.), but is

Peer) reviewing PDF | (2021:08:65264:2:0:NEW 5 Nov 2021) 
307 nonetheless informative because it focuses on one of the tissues primarily involved in body

308 support and propulsion during terrestrial locomotion.

309

310

$311 \# 2$ - Mean size-normalized isometric strength v. $m_{\text {body }}$

312 Following calculation of PCSA as per Equation 1, the arithmetic mean across all muscles was

313 taken. Although PCSA is an estimator of maximal isometric force-generating capacity of a

314 muscle, as a measure of area it is not a particularly intuitive descriptor of force, in and of itself.

315 To present mean PCSA in a more tangible form, it was converted to maximal isometric force in 316 multiples of body weight (BW):

$$
F_{\max } *=\frac{\mathrm{PCSA} \cdot \sigma}{m_{\mathrm{body}} \cdot g}
$$

318

319

320

321

322

323

324

325

326

327

328

329

330

331

332

333

334

335

336

7

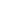

where $\sigma$ is maximal isometric stress, assumed here as 300,000 N/m² (Bates \& Falkingham 2018; Hutchinson 2004; Medler 2002), and $g$ is the acceleration due to gravity. Dividing by body mass means that under isometry the scaling exponent is that of PCSA minus 1.0 (i.e., $-1 / 3$ ), which in turn makes it more straightforward to interpret in the context of scaling. That is, PCSA can scale with positive allometry and yet force-generating capacity can decline with increasing size if $2 / 3<$ PCSA exponent $<1$, whereas the sign of the exponent for $F_{\max } *$ is immediately indicative of whether a strength deficit exists at larger body size: only if it is positive is relative forcegenerating capacity (i.e., relative performance) maintained with increasing size. It should be noted that using a different value for $\sigma$ would not alter the resulting scaling exponent.

$\underline{\# 2 \mathrm{~b}-\text { Median } F_{\max }}{ }^{*}$ v. $m_{\text {body }}$

The relative masses and force-generating capacities of individual limb muscles typically do not follow an even or symmetrical distribution (Table S1), such that the analysis of means as above may be influenced by one or two exceedingly strong, or weak, muscles in the limb. Thus, the median PCSA for all muscles was also computed, and converted to $F_{\max }{ }^{*}$ as per Equation 2. Under isometry, the scaling exponent would also be $-1 / 3$. 
$337 \quad \# 2 \mathrm{c}-$ Total $F_{\max }^{*}$ v. $m_{\text {body }}$

338 In the current dataset there is considerable variation in the number of individually measured and 339 reported muscles for a given species. These differences can reflect investigator judgement in the 340 splitting or grouping of muscle heads for measurement, but can also be due to legitimate

341 anatomical differences between species; for example, crocodylians have a single long digital 342 flexor in the hindlimb, whereas birds have up to six. Variation in the number of muscles may 343 influence the mean or median PCSA, and so to account for this the total PCSA was also 344 computed and converted to $F_{\max }{ }^{*}$ as per Equation 2. Again, under isometry the scaling exponent 345 would be $-1 / 3$.

\#3 - Characteristic fascicle length v. $m_{\text {body }}$

Fascicle length is almost ubiquitously investigated in studies of muscle architecture scaling, yet in a broad comparison across amniotes it is not sensible to investigate fascicle length in and of itself, either for individual muscles, functional groups or for the whole limb. This is due to the great variation that can exist in muscle size, origins, insertions and lengths, as well as differences of subdivision (differentiation) between different taxonomic groups, which can all lead to marked variation in fascicle length irrespective of body size. An alternative approach is to compute a single 'characteristic fascicle length' for the limb as a whole, as the weighted harmonic mean of the fascicle lengths of each individual muscle (cf. Alexander et al. 1981):

$$
L^{*}=\frac{\Sigma m_{\text {muscle }}}{\rho \cdot \Sigma \mathrm{PCSA}} .
$$

358

359

360

361

362

This effectively replaces the musculature of the whole limb (or proximal or distal compartment thereof) with a single equivalent muscle. Note that in using PCSA, Equation 3 weights fascicle lengths by both muscle mass and $\cos \left(\alpha_{0}\right)$, and thus the equivalent whole-limb muscle also factors in pennation. Furthermore, it can be seen that $L^{*}$ is approximately inversely proportional to mean relative (mass-normalized) PCSA (Eng et al. 2008). As for 'real' fascicle length, the scaling exponent under isometry would be $1 / 3$.

$\underline{\#-\Sigma P C S A}$ v. $\Sigma m_{\text {muscle }}$ 
367 By removing the context of $m_{\text {body }}$, this provides a direct assessment of the 'fascicle packing

368 problem', by testing how whole-limb force-generating capacity compares against the amount of 369 available muscle mass. Under isometry, the scaling exponent would be $2 / 3$; note that isometry 370 may reflect scale invariance of pennation angle (which would be theoretically expected; see also 371 Dick and Clemente 2017, Cieri et al. 2020), fascicle length, or a more complex combination of both parameters. Comparison 3 also addresses the fascicle packing problem, but indirectly.

\section{Statistical analyses}

376

All data processing and analyses were conducted in R v.4.1.0 (R Core Team 2021), using the 'caper' (v.1.0.1; Orme et al. 2018), 'evomap' (v 0.0.9; Smaers \& Rohlf 2016) 'phytools’ (v.0.7; Revell 2012), 'geiger' (v.2.0.7; Harmon et al. 2008) and 'nlme' (v. 3.1; Pinheiro et al. 2021) packages; the full set of code and data used are provided in the Supplemental Information. Data were logarithmically transformed (base 10) prior to analysis, facilitating the use of linear statistical models. Phylogenetically informed statistical analyses were conducted using a single, fully resolved, time-calibrated phylogenetic tree of the study taxa (Fig. S1). The tree was generated using the TimeTree database (www.timetree.org; Hedges et al. 2006), which included all taxa except for Isoodon fusciventer; this was substituted with Isoodon obesulus (the only species of Isoodon in the database), which has no effect on divergence times with respect to other taxa in the present study.

For each of the above anatomical comparisons (for whole-limb and proximal and distal compartments), allometric scaling equations of the form $\log _{10} Y=\log _{10} A+B \log _{10} X$ were derived for mammals, reptiles and bipeds separately, using phylogenetic generalized least squares (pGLS or 'phylogenetic regression'; Smaers \& Rohlf 2016) to determine slopes (exponents, $B$ ) and intercepts $\left(\log _{10} A\right)$. This simultaneously estimated the $\lambda$ parameter of Pagel (1999) using maximum likelihood, offering greater flexibility than a strict Brownian motion model of evolution in accounting for phylogenetic signal in the data. Additionally, the $95 \%$ confidence interval (CI) of the slope was determined, using the $t$-distribution, to facilitate comparison 
398

399

400

401

402

403

404

405

406

407

408

409

410

411

412

413

414

415

416

\section{Results}

418

419

420

421

422

423

424

425

426

427

428

\section{$\underline{\text { Results }}$}

scaling was deemed to significantly depart from isometry at the $P=0.05$ level. Subsequently, all groups were collated together and a new pGLS fit was computed to generate a 'pan-amniote' regression for a given anatomical comparison (again for whole-limb and proximal and distal compartments), thus deriving a statistical predictive framework that can be applied to extinct species. In addition to estimator coefficients, 95\% CIs and prediction intervals were also derived (Smaers \& Rohlf 2016), which can provide error margins for future estimations.

To ascertain whether mammals, reptiles or bipeds exhibited different allometric trajectories for a given anatomical comparison, a phylogenetic analysis of covariance (pANCOVA) was performed, which tested for differences in slope and intercept between groups (both separately and together; Smaers \& Rohlf 2016). Here the off-diagonal elements of the variance-covariance matrix were scaled by the $\lambda$ parameter estimated during calculation of the pan-amniote regression, to minimize false negatives caused by an overly conservative assumption of strict Brownian motion. The analyses were also run without accounting for phylogeny (ANCOVA, $\lambda=$ 0 ), to evaluate the effect of phylogenetic relatedness on the results, given that the mammal, reptile and biped samples are nearly mutually exclusive phylogenetically. Statistical significance was set at $P=0.05$.

Scaling results and exponents are presented graphically in Figs 2-5; as the results for mean $F_{\max }{ }^{*}$ (comparison 2) were highly similar to those for median and total $F_{\max }{ }^{*}$ (comparisons $2 \mathrm{~b}$ and $2 \mathrm{c}$, respectively), the latter are presented in the Supplemental Information (SI text, Figs S2, 3). The results for hindlimb analyses excluding the caudofemoralis longus (CFL) from the reptile (i.e., non-avian saurian) dataset are presented in Figs S4-S9. The results of inter-group comparisons of allometries are presented in Tables $2-5$, with those for median and total $F_{\max }{ }^{*}$ presented in the Supplemental Information (Tables S2, 3); the results for hindlimb analyses excluding the CFL from the reptile dataset are presented in Tables S4-S9. Coefficients for the pan-amniote regressions are reported in Table 6, with coefficients for hindlimb analyses excluding the CFL from the reptile dataset presented in Table S10. 


\section{Total muscle mass v. body mass}

432

433 Reptiles almost ubiquitously show negative allometry, whereas mammals and bipeds do not

434

435

436

437

438

439

440

441

442

443

444

445

446

447

450

451

452

453

454

455

456

457

458

459

show significant departure from isometry (Fig. 2). The one exception to this generalization is negative allometry in the distal hindlimb of mammals: larger mammals tend to have relatively lighter (less muscled) distal hindlimbs. When the CFL is excluded, the values of the slopes (and CIs) for reptiles change minimally, but this is sufficient to render the revised scaling statistically indistinguishable from isometry (Fig. S4). Analyses of covariance (without accounting for phylogeny) indicate that mammals, reptiles and bipeds each exhibit significantly different allometric trajectories, in terms of both slope and intercept (Tables 2, S4). Reptiles have a lower slope and intercept compared to mammals, indicating that they have proportionately lower muscle mass, especially at large body sizes. In contrast, bipeds have proportionately greater hindlimb muscle mass than both mammals and reptiles, especially at larger body size and particularly in the distal limb. Many of these differences disappear (i.e., become statistically nonsignificant) once phylogeny is taken into consideration using the pANCOVA

\section{Mean size-normalized isometric strength $\left(F_{\max }{ }^{*}\right) \mathrm{v}$. body mass}

Both mammals and reptiles generally do not show significant departure from isometry, although reptiles exhibit negative allometry in the distal hindlimb (Fig. 3). When the CFL is excluded, the values of the slopes for reptiles change minimally, but narrower CIs render the revised scaling significantly negatively allometric, for both the whole and proximal hindlimb (Fig. S5). Notably, bipeds exhibit strong positive allometry throughout the hindlimb, particularly in the distal limb (Fig. 3); notwithstanding the small sample size, mean size-normalized force-generating capacity appears to be scale-invariant. Analyses of covariance without accounting for phylogeny indicate that mammals, reptiles and bipeds frequently exhibit significantly different allometric trajectories, especially in terms of slope (Tables 3, S5). Differences in intercept were mostly detected for the forelimb, where reptiles exhibit a markedly lower intercept than mammals, 
460 indicating lower mean force-generating capacity regardless of body size. Most of the differences

461 between mammals and reptiles disappear once phylogeny is taken into consideration using

462 pANCOVA; in contrast, most differences between mammals and bipeds, and reptiles and bipeds, 463 were retained following phylogenetic correction, attesting to strong allometric deviation in the 464 biped sample.

465

466

\section{Characteristic fascicle length $\left(L^{*}\right) \mathrm{v}$. body mass}

468

469

470

In the forelimb, both mammals and reptiles exhibit negative allometry, although in reptiles this is not statistically significant in the distal limb, due to wide CIs in that instance (Fig. 4). Mammals and bipeds also exhibit negative allometry in the hindlimb, although in mammals this is driven by the distal limb only; mirroring patterns noted above, bipeds show a stronger departure from isometric scaling in the distal limb. Reptiles do not show any significant departure from isometry in the hindlimb, a result that remains unaltered when the CFL is excluded from the dataset (Fig. S8). In stark contrast to the previous comparisons, ANCOVA (without accounting for phylogeny) revealed almost no significant difference among mammals, reptiles or bipeds (Tables 4, S8). The only difference detected (which disappeared following phylogenetic correction using pANCOVA) was that mammals have a lower intercept in the distal hindlimb compared to both reptiles and bipeds, indicating that, overall, the distal hindlimb of mammals possesses proportionately shorter muscle fascicles.

\section{Total PCSA v. total muscle mass}

Mammals and bipeds almost ubiquitously exhibit positive allometry, whereas reptiles generally do not exhibit statistically significant departures from isometry (Fig. 5). Two exceptions to this are mammals not showing a significant departure from isometry in the distal forelimb, and reptiles exhibiting positive allometry in the forelimb as a whole. Excluding the CFL does not significantly alter the reptile hindlimb scaling patterns (Fig. S9). As for $L^{*}$, ANCOVA (without accounting for phylogeny) notably revealed almost no significant difference among mammals, 
491 reptiles or bipeds (Tables 5, S9). Mammals have a lower slope in the hindlimb overall compared

492 to bipeds, although this cannot be attributed to differences within the proximal or distal

493 compartments. Additionally, mammals have a higher intercept in the distal hindlimb compared

494 to reptiles (a result which disappeared following phylogenetic correction using pANCOVA),

495 indicating that the distal limb of mammals possesses relatively greater fascicle area.

496

497

498

\section{Pan-amniote regression}

499

500

Coefficients for the pan-amniote regressions (Table 6) provide an empirical basis for estimating

501

some important measures of muscle mass and force-generating capacity in extinct terrestrial

502

amniotes. Note that the coefficients reported here were computed to the exclusion of bipeds,

503 given that bipeds have been shown above to frequently differ from quadrupeds, and that the

504

505

506

507

508

509

510

511

512

513

\section{Discussion}

515

516 Through a synthesis of new dissection data with previously published results, the present study

517 aimed to holistically appraise limb muscle scaling in terrestrial amniotes. In particular, it sought

518 to investigate how muscle mass (size) and force-generating capacity (strength) scales at the level

519 of the whole limb, to explore whether this reflects previously observed patterns noted for

520 individual muscles or functional groups across disparate clades, and to assess how tightly

521 constrained amniote limbs are in terms of whole-limb muscular composition. A subsidiary 
522 objective was to generate statistical relationships that have predictive value in inferring function

523 in extinct amniotes. In synthesizing data from numerous studies, it must be acknowledged that

524 the resulting dataset will likely contain a certain level of 'noise' due to various sources. One

525 pertinent source of error is the measurement of fascicle length in fresh specimens: as was the

526 case in the present study, this is typically undertaken following removal of a given muscle from

527 the limb, whereupon the fascicles may non-systematically deviate from a 'reasonable' or

528 functionally relevant length. This problem may be partially mitigated by using formalin-fixed

529 specimens, where the limb joints can be locked in physiologically realistic poses prior to fixation

530 (thus limiting fascicle length change after the muscle is removed), although this approach can

531 involve its own set of challenges, such as muscle shrinkage (Martin et al. 2020; Kikuchi and

532 Kuraoka 2014). Digital methods that permit architectural measurement in situ, such as contrast-

533 enhanced scanning and automated fascicle tracking (e.g., Sullivan et al. 2019), may be able to

534 avoid these issues, but thus far are limited in spatial scale and hence anatomical and taxonomic

535 scope. Additional sources of noise in the present study will also include variation in the

536 approaches of prior studies, in terms of investigator, occasional exclusion of some small

537 muscles, subjective subdivision of muscle complexes and so on. Nonetheless, given the wide

538 diversity and size range of species covered here, this was considered acceptable for the present

539 study's broad scope.

540

541

542

Mass and force-generating capacity scaling across terrestrial amniotes

543

544 In terms of total muscle mass (Fig. 2), the present study found that reptile (i.e., non-avian

545 saurian) limbs typically show negative allometry with respect to body mass (exponents 0.884 to

546 0.939), whereas mammals (exponents 0.989 to 1.019) and bipeds (exponents 1.104 to 1.109, but

547 wide CIs) typically displayed isometry. This is in partial agreement with prior studies, where

548 weak negative to modest positive allometry has been recovered (exponents 0.9 to 1.15 across all

549 amniotes; see references in Introduction). The differences in findings may be due to multiple

550 factors, including variation in sample sizes affecting CI calculation, the line-fitting approach

551 used and even the species that contribute to the underlying datasets. It may also reflect a genuine

552 biological phenomenon, where allometric patterns observed for a select few muscles (i.e., those

Peer] reviewing PDF | (2021:08:65264:2:0:NEW 5 Nov 2021) 
553 which extend the range of exponents recovered by previous studies) are 'cancelled out' by those

554 of many other muscles, when considered together at the level of the whole limb. Irrespective of

555 the proximate cause(s), it is evident that reptiles have proportionately less muscle mass than

556 mammals or bipeds, and bipeds have proportionately greater hindlimb muscle mass, especially in

557 the distal limb. Neither result is hardly surprising, given that the reptiles sampled have short

558 limbs and a long, massive tail that contributes substantially toward total body mass, and that

559 bipeds (by virtue of being bipeds) ought to invest a greater fraction of body mass into longer,

560 more heavily muscled hindlimbs.

561

562 Muscle force-generating capacity in the present study was expressed in a more intuitive fashion

563 by normalizing to units of body weight. Thus, to compare exponents derived here to those of

564 prior studies that have examined PCSA directly, this requires adding or subtracting 1.0 to the

565 exponent. Three different measures of normalized force-generating capacity $\left(F_{\max }{ }^{*}\right)$ were

566 explored here - mean (Fig. 3), median (Fig. S2) and total (Fig. S3) - which showed a consistent

567 overarching set of patterns with respect to body mass. Mammals and reptiles overall exhibit

568 isometric scaling, although in some circumstances mammals tended towards positive allometry

569 (total range of exponents -0.30 to -0.245 ) whereas reptiles tended towards negative allometry

570 (total range of exponents -0.465 to -0.302). This is partially consistent with the results of prior

571 studies (corrected exponents -0.31 to -0.09 ; see references in Introduction). Again, discrepancies

572 in findings may belie the different spatial scales concerned; for example, the high end of

573 previously reported exponents derive from the plantaris muscle of the mammalian hindlimb

574 (Pollock \& Shadwick 1994) and the proximal forelimb of felids (Cuff et al. 2016a). Of note,

575 reptiles often displayed a markedly lower intercept than mammals or bipeds, indicative of

576 reduced relative force-generating capacity and again consistent with possessing short limbs and

577 massive tails. It therefore follows that how 'body mass' is gauged (total body mass, body mass

578 excluding tail, etc.) will influence interpretations of interspecific differences in force-generating

579 capacity, although from a purely mechanical perspective total body mass is appropriate in the

580 context of terrestrial locomotion, since ultimately the limbs must support and propel all of it.

581

582 Bipeds were found to almost ubiquitously display positive allometry in muscle force-generating 583 capacity, and were frequently found to significantly differ from mammals and reptiles even after 
584 phylogeny was accounted for (in 13 of 15 comparisons with mammals, all comparisons with 585 reptiles); again, this can be related to the greater investment of biomass in the hindlimbs of these 586 species. Notably, positive allometry was exceedingly strong in the distal hindlimb (total range of 587 exponents -0.016 to 0.025$)$, to the point that relative force-generating ability may increase with 588 increasing body size. This is comparable to exponents reported for bipedal hopping macropods 589 (corrected exponents -0.22 to 0.33; Bennett \& Taylor 1995; McGowan et al. 2008), although 590 intriguingly is higher than the exponents recovered by Maloiy et al. (1979) for the ankle 591 extensors of a selection of ground-dwelling birds (corrected exponent -0.24 to -0.19 ). These 592 results should be viewed with some caution, given that only six species were examined in the 593 present study. Nonetheless, they raise the interesting question of whether extreme positive 594 allometry would persist at larger body sizes beyond the largest extant biped, the ostrich (Struthio 595 camelus). For example, how would muscle strength fare in multi-tonne non-avian theropod dinosaurs, and what implications may this have for locomotor performance? These are questions that must await future investigation, but the comparative dataset marshalled here can provide an empirical basis for such efforts.

599

600

601

In lieu of investigating fascicle length per se, this study examined an equivalent region-level metric, characteristic fascicle length $\left(L^{*}\right)$, which tended to exhibit negative allometry with 602 respect to body mass in both mammals and bipeds, as well as the forelimb of reptiles. The 603 exponents obtained ( 0.171 to 0.324$)$ fell within the range reported previously for actual fascicle 604 length in individual muscles or functional groups ( 0.14 to 0.5 ; see references in Introduction). Negative allometry indicates that muscle fascicles are on the whole proportionately shorter in larger species. This is especially true in the mammalian distal hindlimb, which also exhibits a lower intercept (Fig. 4); shorter muscle fascicles in the distal hindlimb of mammals in turn translate to relatively greater PCSA per unit mass in this compartment (Fig. 5). All else being equal, proportionately shorter muscle fascicles in larger species will translate to reduced joint excursion, and at least for mammals and birds this correlates with the habitual use of more extended limb postures in larger species (Biewener 1989; Biewener 2005; Bishop et al. 2018;

612 Gatesy \& Biewener 1991). 


\section{Fascicle packing}

617

618 Analysis of covariance of muscle mass and force-generating capacity identified numerous

619 differences in scaling patterns between mammals, reptiles and bipeds, but when phylogeny was

620 taken into account the majority of these became statistically non-significant (Tables 2 ,3, S2, S3).

621 Hence there are genuine physical differences in mass or force-generating capacity between each

622 group (which is biomechanically relevant), and these largely exist due to each group having

623 evolved along different phylogenetic trajectories. However, when total muscle mass was factored

624 into consideration, either directly (comparison 4: $\Sigma$ PCSA v. $\Sigma m_{\text {muscle }}$ ) or indirectly (comparison

625 3: $L^{*}$ v. $m_{\text {body }}$, wherein $\Sigma m_{\text {muscle }}$ is used to compute $\left.L^{*}\right)$, ANCOVA recovered almost no

626 statistically significant difference between any group; this was true regardless of whether

627 phylogeny was accounted for or not, and was observed for the limb as a whole as well as

628 proximal and distal compartments separately (Tables 4, 5). That is, the fore- and hindlimbs of

629 reptiles, mammals and bipeds almost ubiquitously follow a single common scaling pattern ${ }^{1}$. This

630 in turn implies that observed differences in whole-limb force-generating capacity between

631 groups are principally driven by differences in limb muscle mass, not internal architecture: one

632 group does not 'pack their fascicles better' for a given volume of limb muscle, they just invest a

633 greater fraction of total biomass into limb muscle in the first instance. The only possible exception to this generality is the distal hindlimb of mammals, which at larger body sizes at least do indeed appear to 'pack their fascicles better', with a greater PCSA (and in turn, forcegenerating capacity) per unit muscle mass, correlating with proportionately shorter fascicles for a given body size. All else being equal, this result suggests that larger species may benefit from greater elastic energy storage in the distal hindlimb tendons during locomotion (cf. Pollock \& Shadwick 1994), although it should nevertheless be treated with caution, since a large proportion of the currently sampled mammals are 'cursorial' taxa, which may bias the analysis.

\footnotetext{
${ }^{1}$ This might at first seem at odds with the finding that, for example, mammals exhibit positive allometry whereas reptiles do not show a significant departure from isometry. The apparent contradiction can be reconciled by noting that these comparisons are testing two different things in isolation of one another, whereas in reality there is a gradation across successively overlapping exponents and CIs - the statistical analogue of a 'ring species'.
} 
642 The existence of a single overarching pattern, across a diverse array of terrestrial amniotes that

643 span more than four orders of magnitude in body mass, is remarkable. It implies the tendency 644 towards some adaptive optimum in organismal 'design' or, more likely, the presence of one or 645 more constraints that prevent significant and systematic deviation from a common pattern. These 646 constraints may be functional in nature, such as trade-offs that could occur between conflicting 647 requirements of individual muscles in the execution of disparate tasks (see Introduction), or may 648 have a developmental basis (e.g., Evans et al. 2021). Given the multidimensional and nonlinear 649 aspects of muscle architecture and function, and terrestrial locomotor biomechanics in general, it 650 would be naïve to suggest that a bivariate statistical model such as those derived here can 651 sufficiently represent the mechanical phenomena involved (Taylor \& Thomas 2014).

652 Deciphering the proximate underlying cause(s) of this strong consistency must therefore await 653 future study. It is also important to recognize that there is still scope for (non-systematic) 654 variation; for example, the greyhound (Canis familiaris, $27 \mathrm{~kg}$ ) and snow leopard (Panthera 655 uncia, $36 \mathrm{~kg})$ have near-identical total hindlimb muscle mass $(\sim 1.64 \mathrm{~kg})$, yet the greyhound 656 which is selectively bred for high-speed running - has approximately double the PCSA of the 657 snow leopard ( 0.028 v. $0.014 \mathrm{~m}^{2}$; Table S1). Important insight into the fascicle packing problem 658 can therefore be gained by better understanding the reasons for variation about the common pan659 amniote pattern, through exploring variables that were not investigated in the current analyses, 660 such as segment lengths, muscle moment arms, joint mobility, posture and locomotor ecology.

\section{Considerations for future studies}

664

665 A number of other points are worth noting in the context of future comparative investigations of 666 muscle architecture. Firstly, despite a superficially broad taxonomic coverage, the dataset of the

667 present study is still biased, with few birds, marsupials or non-varanid squamates, numerous 668 'cursorial' eutherians and not one testudine; future studies should therefore target currently 669 under- or unsampled parts of amniote phylogeny. Increased diversity will be especially useful for 670 elucidating the true extent, and underlying cause(s) of, conservatism in whole-limb fascicle 671 packing. Furthermore, it is recommended that as much architectural data be collected and 672 reported as possible, even if it may not all be immediately used in a given study, since this will 
673 maximize its potential utility for later studies. One reason why several prior datasets were

674 excluded from the present study was their omission of pennation angle from the set of reported

675 (or even collected) measurements. A second point worth considering is that, out of necessity, the

676 present study ignored the intrinsic musculature of the manus and pes; if further datasets for these

677 muscles become available in the future, they should be analysed, since this may reveal important

678 differences between plantigrade and digitigrade species.

679

680 One final consideration is that the present study found numerous differences between mammals, 681 reptiles and bipeds in the allometry of muscle mass and force-generating capacity with respect to 682 body mass. This is hardly surprising, but it has an important implication for how comparative 683 studies should undertake their analyses. In order to facilitate comparison within or across 684 species, previous studies that have explored muscle architecture and function typically normalize 685 raw architectural measurements by body mass, or the dimensionally appropriate exponent of 686 body mass such as $m_{\text {body }}{ }^{1 / 3}$ for normalized fascicle length (Allen et al. 2010; Bates \& Schachner 687 2012; Dick \& Clemente 2016; Fahn-Lai et al. 2020; Martin et al. 2019; Regnault et al. 2020). 688 This may be acceptable for comparisons of closely related species, but becomes questionable 689 when divergent allometries with body mass are involved. A more sensible approach for future 690 studies would be to normalize against more directly relevant parameters, such as limb or limb 691 segment length, or alternatively by the appropriate clade-specific scaling exponents. Exactly 692 which is the most appropriate normalizing metric to use may vary depending on a given study's 693 core questions, and deserves further scrutiny.

694

695

696 Conclusion

697

698 Whole-limb scaling of muscle mass and force-generating capacity generally follows the same 699 patterns reported previously for individual muscles or functional groups of muscles, although 700 some instances of tendency towards isometry (as opposed to previously reported positive or 701 negative allometry) were noted. This is the first time that muscle scaling has been addressed at 702 such a broad spatial scale, across a diverse array of terrestrial amniotes. Additionally, some 703 important differences were observed between proximal and distal limb compartments, 
704 particularly in the hindlimb of mammals and bipeds, which may reflect the 'cursorial' habits of 705 many of the species investigated here. The mammalian distal hindlimb has proportionately less 706 muscle mass and shorter fascicles, but per unit muscle mass has a higher PCSA; and the distal 707 hindlimb of bipeds has proportionately greater muscle mass and PCSA.

708

709

710

711

712

713

714

715

716

717

718

719

720

721

722

723

724

725

726

727

728

729

730

731

732

733

Almost all differences in force-generating capacity between groups appear to be due principally to differences in muscle mass, rather than muscle architecture. Thus, one group does not systematically 'pack their fascicles better' than another, instead they simply invest more biomass into limb muscle. The underlying reason(s) for a single overarching relationship across extant amniotes remains to be determined, although it echoes conservatism in other aspects of musculoskeletal design, such as tissue mechanical properties.

A comparative dataset of extant amniotes spanning almost five orders of magnitude in body size has been assembled, which can be built upon and used by future studies in the analysis of muscle architecture diversity. This dataset also forms the basis for a suite of pan-amniote predictive equations that can be used to estimate bulk muscle mass and force-generating capacity, along with error margins, for extinct species. These can be used if estimates of body mass are available, or alternatively from estimates of total limb muscle volume, as derived from digital volumetric reconstructions based on skeletal material. Reliably inferring the force-generating capacity of individual muscles in extinct species remains elusive (Bishop et al. 2021a), but inferences of total limb muscle force-generating capacity provides a step closer toward achieving this goal.

\section{$\underline{\text { Acknowledgements }}$}

T. French (Massachusetts Division of Fisheries and Wildlife) and R. Reed (United States Geological Survey, Daniel Beard Center) are thanked for providing the opossum and tegu specimens dissected here; P. Bergmann, C. Clemente, S. Cox, M. Martin and J. Rubenson are thanked for providing the original architectural data from their prior studies; and J. Hughes is 
734 thanked for logistical assistance with dissections. The constructive comments of two anonymous

735

736

737

738

739

740

741

742

743

744

745

746

747

748

749

750

751

752

753

754

755

756

757

758

759

760

761

762

763

764

765

766

767

768

769

770

771

772

773

774

775

776

reviewers, and the editor, on a prior version of the manuscript are also greatly appreciated.

\section{Author contributions}

P.J.B. and S.E.P. conceived the study design; P.J.B. collected anatomical data; P.J.B. and

M.A.W. conducted statistical analyses and initial interpretations; all authors contributed to final interpretations and manuscript writing.

\section{$\underline{\text { References }}$}

Alexander RM. 1977. Allometry of the limbs of antelopes (Bovidae). Journal of Zoology 183:125-146.

Alexander RM, Jayes AS, Maloiy GMO, and Wathuta EM. 1981. Allometry of the leg muscles of mammals. Journal of Zoology 194:539-552.

Allen V, Elsey RM, Jones N, Wright J, and Hutchinson JR. 2010. Functional specialization and ontogenetic scaling of limb anatomy in Alligator mississippiensis. Journal of Anatomy 216:423445.

Allen V, Molnar J, Parker W, Pollard A, Nolan G, and Hutchinson JR. 2015. Comparative architectural properties of limb muscles in Crocodylidae and Alligatoridae and their relevance to divergent use of asymmetrical gaits in extant Crocodylia. Journal of Anatomy 225:569-582.

Bates KT, and Falkingham PL. 2018. The importance of muscle architecture in biomechanical reconstructions of extinct animals: a case study using Tyrannosaurus rex. Journal of Anatomy 233:625-635.

Bates KT, and Schachner ER. 2012. Disparity and convergence in bipedal archosaur locomotion. Journal of the Royal Society Interface 9:1339-1353.

Bennett MB. 1996. Allometry of the leg muscles of birds. Journal of Zoology 238:435-443.

Bennett MB, and Taylor GC. 1995. Scaling of elastic strain energy in kangaroos and the benefits of being big. Nature 378:56-59.

Bergmann PJ, and Hare-Drubka M. 2015. Hindlimb muscle anatomical mechanical advantage differs among joints and stride phases in basilisk lizards. Zoology 118:291-298.

Biewener AA. 1989. Scaling Body Support in Mammals: Limb Posture and Muscle Mechanics. Science 245:45-48.

Biewener AA. 2005. Biomechanical consequences of scaling. Journal of Experimental Biology 208:16651676.

Biewener AA, and Full RJ. 1992. Force platform and kinematic analysis. In: Biewener AA, ed. Biomechanics - Structures and Systems: A Practical Approach. New York: Oxford University Press, 45-73.

Bishop PJ. 2015. A critical re-evaluation of the hindlimb myology of moa (Aves: Dinornithiformes). Memoirs of the Queensland Museum 59:187-246.

Bishop PJ, Cuff AR, and Hutchinson JR. 2021a. How to build a dinosaur: musculoskeletal modelling and simulation of locomotor biomechanics in extinct animals. Paleobiology 47:1-38.

10.1017/pab.2020.46

Peer] reviewing PDF | (2021:08:65264:2:0:NEW 5 Nov 2021) 
777

778

779

780

781

782

783

784

785

786

787

788

789

790

791

792

793

794

795

796

797

798

799

800

801

802

803

804

805

806

807

808

809

810

811

812

813

814

815

816

817

818

819

820

821

822

823

824

825

Bishop PJ, Graham DF, Lamas LP, Hutchinson JR, Rubenson J, Hancock JA, Wilson RS, Hocknull SA, Barrett RS, Lloyd DG, and Clemente CJ. 2018. The Influence of Speed and Size on Avian Terrestrial Locomotor Biomechanics: Predicting Locomotion in Extinct Theropod Dinosaurs. PLOS ONE 13:e0192172.

Bishop PJ, Michel KB, Falisse A, Cuff AR, Allen VR, De Groote F, and Hutchinson JR. 2021b. Computational modelling of muscle fibre operating ranges in the hindlimb of a small ground bird (Eudromia elegans), with implications for modelling locomotion in extinct species. PLOS Computational Biology 17:1008843. 10.1371/journal.pcbi.1008843

Bodine SC, Roy RR, Meadows DA, Zernicke RF, Sacks RD, Fournier M, and Edgerton VR. 1982. Architectural, Histochemical, and Contractile Characteristics of a Unique Biarticular Muscle: the Cat Semitendinosus. Journal of Neurophysiology 48:192-201.

Böhmer C, Fabre A-C, Herbin M, Peigné S, and Herrel A. 2018. Anatomical Basis of Differences in Locomotor Behavior in Martens: A Comparison of the Forelimb Musculature Between Two Sympatric Species of Martes. The Anatomical Record 301:449-472.

Calow LJ, and Alexander RM. 1973. A mechanical analysis of a hind leg of a frog (Rana temporaria). Journal of Zoology 171:293-321.

Carlson KJ. 2006. Muscle architecture of the common chimpanzee (Pan troglodytes): perspectives for investigating chimpanzee behavior. Primates 47:218-229.

Carrano MT, and Hutchinson JR. 2002. Pelvic and hindlimb musculature of Tyrannosaurus rex (Dinosauria: Theropoda). Journal of Morphology 253:207-228.

Charles JP, Cappellari O, Spence AJ, Hutchinson JR, and Wells DJ. 2016. Musculoskeletal Geometry, Muscle Architecture and Functional Specialisations of the Mouse Hindlimb. PLoS ONE 11:e147669.

Cieri RL, Dick TJM, and Clemente CJ. 2020. Monitoring muscle over three orders of magnitude: widespread positive allometry among locomotor and body support musculature in the pectoral girdle of varanid lizards (Varanidae). Journal of Anatomy 237:1114-1135.

Cox SM, Easton KL, Lear MC, Marsh RL, Delp SL, and Rubenson J. 2019. The Interaction of Compliance and Activation on the Force-Length Operating Range and Force Generating Capacity of Skeletal Muscle: A Computational Study Using a Guinea Fowl Musculoskeletal Model. Integrative and Comparative Biology.

Cuff AR, Sparkes EL, Randau M, Pierce SE, Kitchener AC, Goswami A, and Hutchinson JR. 2016a. The scaling of postcranial muscles in cats (Felidae) I: forelimb, cervical, and thoracic muscles. Journal of Anatomy 229:128-141.

Cuff AR, Sparkes EL, Randau M, Pierce SE, Kitchener AC, Goswami A, and Hutchinson JR. 2016b. The scaling of postcranial muscles in cats (Felidae) II: hindlimb and lumbosacral muscles. Journal of Anatomy 229:142-152.

Currey JD. 2002. Bones: Structure and Mechanics. Princeton: Princeton University Press.

Dick TJM, and Clemente CJ. 2016. How to build your dragon: scaling of muscle architecture from the world's smallest to the world's largest monitor lizard. Frontiers in Zoology 13:8.

Dick TJM, and Clemente CJ. 2017. Where have All the Giants Gone? How Animals Deal with the Problem of Size. PLOS Biology 15:e2000473.

Ellis RG, Rankin JW, and Hutchinson JR. 2018. Limb Kinematics, Kinetics and Muscle Dynamics During the Sit-to-Stand Transition in Greyhounds. Frontiers in Bioengineering and Biotechnology 6:162.

Eng CM, Smallwood LH, Rainiero MP, Lahey M, Ward SR, and Lieber RL. 2008. Scaling of muscle architecture and fiber types in the rat hindlimb. Journal of Experimental Biology 211:2336-2345.

Etienne C, Houssaye A, and Hutchinson JR. 2021. Limb myology and muscle architecture of the Indian rhinoceros Rhinoceros unicornis and the white rhinoceros Ceratotherium simum (Mammalia: Rhinocerotidae). PeerJ 9:e11314.

Peer] reviewing PDF | (2021:08:65264:2:0:NEW 5 Nov 2021) 
826

827

828

829

830

831

832

833

834

835

836

837

838

839

840

841

842

843

844

845

846

847

848

849

850

851

852

853

854

855

856

857

858

859

860

861

862

863

864

865

866

867

868

869

870

871

872

873

874

875

876
Evans AR, Pollock TI, Cleuren SGC, Parker WMG, Richards HL, Garland KLS, Fitzgerald EMG, Wilson TE, Hocking DP, and Adams JW. 2021. A universal power law for modelling the growth and form of teeth, claws, horns, thorns, beaks, and shells. BMC Biology 19:58.

Fahn-Lai P, Biewener AA, and Pierce SE. 2020. Broad similarities in shoulder muscle architecture and organization across two amniotes: implications for reconstructing non-mammalian synapsids. PeerJ 8:8556.

Gans C, and Bock WJ. 1965. The functional significance of muscle architecture - a theoretical analysis. Ergebnisse der Anatomie und Entwicklungsgeschichte 38.

Gatesy SM, and Biewener AA. 1991. Bipedal locomotion: effects of speed, size and limb posture in birds and humans. Journal of Zoology 224:127-147.

Gaunt AS, and Gans C. 1992. Serially arranged myofibers: An unappreciated variant in muscle architecture. Experientia 48:864-868.

Grand TI. 1977. Body Weight: Its Relation to Tissue Composition, Segment Distribution, and Motor Function. I. Interspecific Comparisons. American Journal of Physical Anthropology 47:211-240.

Harmon LJ, Weir JT, Brock CD, Glor RE, and Challenger W. 2008. GEIGER: investigating evolutionary radiations. Bioinformatics 24:129-131.

Hedges SB, Dudley J, and Kumar S. 2006. TimeTree: A public knowledge-base of divergence times among organisms. Bioinformatics 22:2971-2972.

Hudson PE, Corr SA, Payne-Davis RC, Clancy SN, Lane E, and Wilson AM. 2011a. Functional anatomy of the cheetah (Acinonyx jubatus) forelimb. Journal of Anatomy 218:375-385.

Hudson PE, Corr SA, Payne-Davis RC, Clancy SN, Lane E, and Wilson AM. 2011b. Functional anatomy of the cheetah (Acinonyx jubatus) hindlimb. Journal of Anatomy 218:363-374.

Hutchinson JR. 2004. Biomechanical Modeling and Sensitivity Analysis of Bipedal Running Ability. I. Extant Taxa. Journal of Morphology 262:421-440.

Hutchinson JR, Rankin JW, Rubenson J, Rosenbluth KH, Siston RA, and Delp SL. 2015. Musculoskeletal modelling of an ostrich (Struthio camelus) pelvic limb: influence of limb orientation of muscular capacity during locomotion. PeerJ 3:e1001.

Infantolino BW, Neuberger T, and Challis JH. 2012. The Arrangement of Fascicles in Whole Muscle. The Anatomical Record 295:1174-1180.

Jenkins FA, Jr, and Weijs WA. 1979. The functional anatomy of the shoulder in the Virginia opossum (Didelphis virginiana). Journal of Zoology 188:379-410.

Kikuchi, Y, and Kuraoka, A. 2014. Differences in muscle dimensional parameters btween non-formalinfixed (freeze-thawed) and formalin-fixed specimen in gorilla (Gorilla gorilla). Mammal Study 39:65-72.

Lamas LP, Main RP, and Hutchinson JR. 2014. Ontogenetic scaling patterns and functional anatomy of the pelvic limb musculature in emus (Dromaius novaehollandiae). PeerJ 2:e716.

Lautenschlager S, Gill PG, Luo Z-X, Fagan MJ, and Rayfield EJ. 2018. The role of miniaturization in the evolution of the mammalian jaw and middle ear. Nature 561:533-537.

Maloiy GMO, Alexander RM, Njau R, and Jayes AS. 1979. Allometry of the legs of running birds. Journal of Zoology 187:161-167.

Martin ML, Travouillon KJ, Fleming PA, and Warburton NM. 2020. Review of the methods used for calculating physiological cross-sectional area (PCSA) for ecological questions. Journal of Morphology 281:778-789.

Martin ML, Warburton NM, Travouillon KJ, and Fleming PA. 2019. Mechanical similarity across ontogeny of digging muscles in an Australian marsupial (Isoodon fusciventer). Journal of Morphology 280:423-435.

McGowan CP, Skinner J, and Biewener AA. 2008. Hind limb scaling of kangaroos and wallabies (superfamily Macropodoidea): implications for hopping performance, safety factor and elastic savings. Journal of Anatomy 212:153-163.

Medler S. 2002. Comparative trends in shortening velocity and force production in skeletal muscle. American Journal of Physiology 283:R368-R378. 
877

878

879

880

881

882

883

884

885

886

887

888

889

890

891

892

893

894

895

896

897

898

899

900

901

902

903

904

905

906

907

908

909

910

911

912

913

914

915

916

917

918

919

920

921

922

923

924

925

926

927

Mendez J, and Keys A. 1960. Density and composition of mammalian muscle. Metabolism - Clinical and Experimental 9:184-188.

Moore AL, Budny JE, Russell AP, and Butcher MT. 2012. Architectural Specialization of the Intrinsic Thoracic Limb Musculature of the American Badger (Taxidea taxus). Journal of Morphology 274:35-48.

Ogihara N, Makishima H, Aoi S, Sugimoto Y, Tsuchiya K, and Nakatsukasa M. 2009. Development of an Anatomical Based Whole-Body Musculoskeletal Model of the Japanese Macaque (Macaca fuscata). American Journal of Physical Anthropology 139:323-338.

Olson RA, Glenn ZD, Cliffe RN, and Butcher MT. 2018. Architectural Properties of Sloth Forelimb Muscles (Pilosa: Bradypodidae). Journal of Mammalian Evolution 25:573-588.

Olson RA, Womble MD, Thomas DR, Glenn ZD, and Butcher MT. 2016. Functional Morphology of the Forelimb of the Nine-Banded Armadillo (Dasypus novemcinctus): Comparative Perspectives on the Myology of the Dasypodidae. Journal of Mammalian Evolution 23:49-69.

Orme D, Freckleton R, Thomas G, Petzoldt T, Fritz S, Isaac N, and Pearse W. 2018. caper: Comparative Analyses of Phylogenetics and Evolution in R.

Pagel M. 1999. Inferring the historical patterns of biological evolution. Nature 401.

Paxton H, Anthony NB, Corr SA, and Hutchinson JR. 2010. The effects of selective breeding on the architectural properties of the pelvic limb in broiler chickens: a comparative study across modern and ancestral populations. Journal of Anatomy 217:153-166.

Payne RC, Crompton RH, Isler K, Savage R, Vereecke EE, Günther MM, Thorpe SKS, and D'Août K. 2006. Morphological analysis of the hindlimb in apes and humans. I. Muscle architecture. Journal of Anatomy 208:709-724.

Payne RC, Hutchinson JR, Robilliard JJ, Smith NC, and Wilson AM. 2005. Functional specialisation of pelvic limb anatomy in horses (Equus caballus). Journal of Anatomy 206:557-574.

Pinheiro J, Bates D, DebRoy S, and Sarkar D. 2021. nlme: Linear and Nonlinear Mixed Effects Models.

Pollock CM, and Shadwick RE. 1994. Allometry of muscle, tendon, and elastic energy storage capacity in mammals. American Journal of Physiology 266:R1022-R1031.

Powell PL, Roy RR, Kanim P, Bello MA, and Edgerton VR. 1984. Predictability of skeletal muscle tension from architectural determinations in guinea pig hindlimbs. Journal of Applied Physiology 57:1715-1721.

R Core Team. 2021. R: a language and environment for statistical computing. Vienna: R Foundation for Statistical Computing.

Rajagopal A, Dembia CL, DeMers MS, Delp DD, Hicks JL, and Delp SL. 2016. Full-Body Musculoskeletal Model for Muscle-Driven Simulation of Human Gait. IEEE Transactions of Biomedical Engineering 63:2068-2079.

Regnault S, Fahn-Lai P, Norris RM, and Pierce SE. 2020. Shoulder Muscle Architecture in the Echidna (Monotremata: Tachyglossus aculeatus) Indicates Conserved Functional Properties. Journal of Mammalian Evolution. 10.1007/s10914-020-09498-6

Revell LJ. 2012. phytools: an R package for phylogenetic comparative biology (and other things). Methods in Ecology and Evolution 3:217-223.

Rose JA, Sandefur M, Huskey S, Demler JL, and Butcher MT. 2013. Muscle Architecture and Out-Force Potential of the Thoracic Limb in the Eastern Mole (Scalopus aquaticus). Journal of Morphology 274:1277-1287.

Rupert JE, Rose JA, Organ JM, and Butcher MT. 2015. Forelimb muscle architecture and myosin isoform composition in the groundhog (Marmota monax). Journal of Experimental Biology 218:194-205.

Sacks RD, and Roy RR. 1982. Architecture of the Hind Limb Muscles of Cats: Functional Significance. Journal of Morphology 173:185-195.

Schmidt-Nielsen K. 1985. Scaling: Why is Animal Size So Important? Cambridge: Cambridge University Press.

Sellers WI, Margetts L, Coria RA, and Manning PL. 2013. March of the Titans: The Locomotor Capabilities of Sauropod Dinosaurs. PLoS ONE 8:e78733.

Peer] reviewing PDF | (2021:08:65264:2:0:NEW 5 Nov 2021) 
928

Smaers JB, and Rohlf FJ. 2016. Testing species' deviation from allometric predictions using the phylogenetic regression. Evolution 70:1145-1149.

Smith NC, Wilson AM, Jespers KJ, and Payne RC. 2006. Muscle architecture and functional anatomy of the pelvic limb of the ostrich (Struthio camelus). Journal of Anatomy 209:765-779.

Smith RJ. 1980. Rethinking allometry. Journal of Theoretical Biology 87:97-111.

Sullivan SP, McGechie FR, Middleton KM, and Holliday CM. 2019. 3D Muscle Architecture of the Pectoral Muscles of European Starling (Sturnis vulgaris). Integrative Organismal Biology 1:oby010.

Taylor GK, and Thomas ALR. 2014. Evolutionary Biomechanics - Selection, Phylogeny, and Constraint. Oxford: Oxford University Press.

Vogel S. 2003. Comparative Biomechanics: Life's Physical World. Princeton: Princeton University Press.

Wareing K, Tickle PG, Stokkan K-A, Codd JR, and Sellers WI. 2011. The muscukoskeletal anatomy of the reindeer (Rangifer tarandus): fore- and hindlimb. Polar Biology 34:1571-1578.

Williams SB, Payne RC, and Wilson AM. 2007a. Functional specialisation of the pelvic limb of the hare (Lepus europeus). Journal of Anatomy 210:472-490.

Williams SB, Wilson AM, and Payne RC. 2007b. Functional specialisation of the thoracic limb of the hare (Lepus europeus). Journal of Anatomy 210:491-505.

Williams SB, Wilson AM, Peckham K, and Payne RC. 2008. Functional anatomy and muscle moment arms of the thoracic limb of an elite sprinting athlete: the racing greyhound (Canis familiaris). Journal of Anatomy 213:373-382.

Wilson A, and Lichtwark G. 2011. The anatomical arrangement of muscle and tendon enhances limb versatility and locomotor performance. Philosophical Transactions of the Royal Society of London, Series B 366:1540-1553.

Witmer LM. 1995. The extant phylogenetic bracket and the importance of reconstructing soft tissues in fossils. In: Thomason JJ, ed. Functional Morphology in Vertebrate Paleontology. Cambridge: Cambridge University Press, 19-33.

Zajac FE. 1989. Muscle and tendon: properties, models, scaling, and application to biomechanics and motor control. Critical Reviews in Biomedical Engineering 17:359-410.

Zihlman AL, McFarland RK, and Underwood CE. 2011. Functional Anatomy and Adaptation of Male Gorillas (Gorilla gorilla gorilla) with Comparison to Male Orangutans (Pongo pygmaeus). The Anatomical Record 294:1842-1855.

(1)

(1)

(1)

(1)

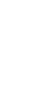

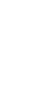

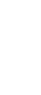

(1)

1

\footnotetext{
Peer] reviewing PDF | (2021:08:65264:2:0:NEW 5 Nov 2021)
} 
Figure captions

979

980

981

982

Figure 1. The requirements on muscles as part of a single, functionally integrated whole may impose constraints on their construction. (A) Changes in the size of one muscle may 983 necessitate change in the size of adjacent muscle, such that total muscle volume (illustrated here in cross-section) may remain relatively constant. (B) Functional specialization of one muscle's 984 architecture may necessitate concomitant changes to the architecture of other muscles, in order 985 for a limb to remain capable of effectively executing a diverse range of tasks; total physiological 986 cross-sectional area (PCSA) may therefore remain relatively constant. In this example, moving from left to right muscle 1 decreases PCSA but muscle 2 increases PCSA, such that total PCSA remains unaltered.

989

Figure 2. Total muscle mass v. body mass across extant terrestrial amniotes. This is shown for the forelimb and hindlimb, in terms of the limb as a whole, and parsed by proximal and distal 992 compartments. Results are plotted on logarithmic coordinates, along with phylogenetic regression (pGLS) and 95\% confidence intervals (CIs). Red $=$ mammals, blue $=$ reptiles, green $=$ bipeds. Note the difference in vertical and horizontal scales. The slope expected under isometry is indicated in each case by the solid grey line, plotted clear of the data for ease of viewing. The slope and 95\% CIs for each group are reported in each case, as are the sample sizes (in parentheses); also indicated are instances where a particular group exhibits statistically significant positive (仓) or negative $(\preceq)$ allometry.

Figure 3. Mean size-normalized isometric strength v. body mass across extant terrestrial amniotes. Conventions as in Fig. 2. See Figs S2 and S3 for the results of median and total sizenormalized isometric strength $\mathrm{v}$. body mass. 
1004 Figure 4. Characteristic fibre length v. body mass across extant terrestrial amniotes.

1005 Conventions as in Fig. 2.

1006

1007 Figure 5. Total physiological cross-sectional area v. total muscle mass across extant

1008 terrestrial amniotes. Conventions as in Fig. 2. 


\section{Figure 1}

The requirements on muscles as part of a single, functionally integrated whole may impose constraints on their construction.

(A) Changes in the size of one muscle may necessitate change in the size of adjacent muscle, such that total muscle volume (illustrated here in cross-section) may remain relatively constant. (B) Functional specialization of one muscle's architecture may necessitate concomitant changes to the architecture of other muscles, in order for a limb to remain capable of effectively executing a diverse range of tasks; total physiological cross-sectional area (PCSA) may therefore remain relatively constant. In this example, moving from left to right muscle 1 decreases PCSA but muscle 2 increases PCSA, such that total PCSA remains unaltered. 


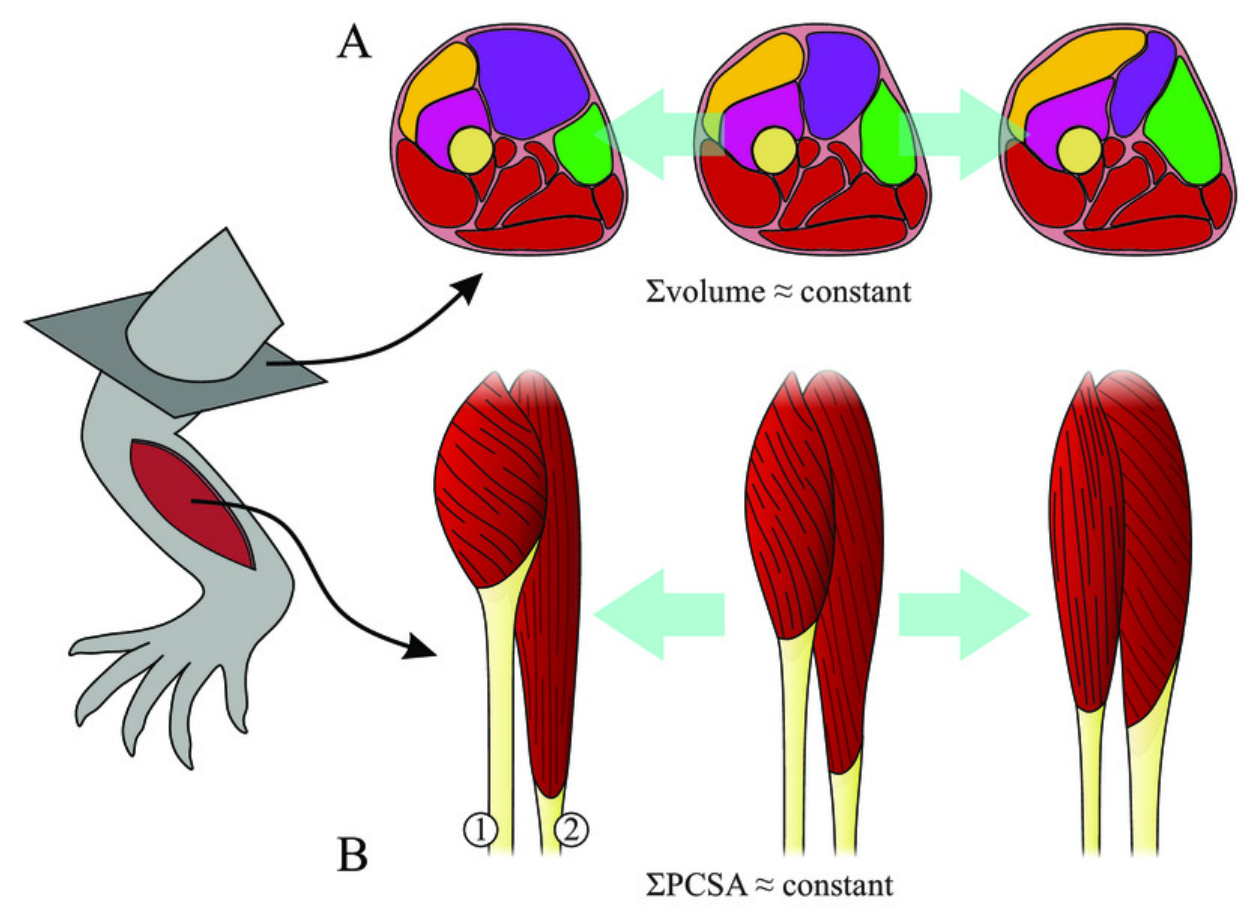




\section{Figure 2}

Total muscle mass v. body mass across extant terrestrial amniotes.

This is shown for the forelimb and hindlimb, in terms of the limb as a whole, and parsed by proximal and distal compartments. Results are plotted on logarithmic coordinates, along with phylogenetic regression (pGLS) and 95\% confidence intervals (Cls). Red $=$ mammals, blue $=$ reptiles, green $=$ bipeds. Note the difference in vertical and horizontal scales. The slope expected under isometry is indicated in each case by the solid grey line, plotted clear of the data for ease of viewing. The slope and $95 \% \mathrm{Cls}$ for each group are reported in each case, as are the sample sizes (in parentheses); also indicated are instances where a particular group exhibits statistically significant positive ( () ) or negative $(\sqrt{)}$ ) allometry. 
forelimb
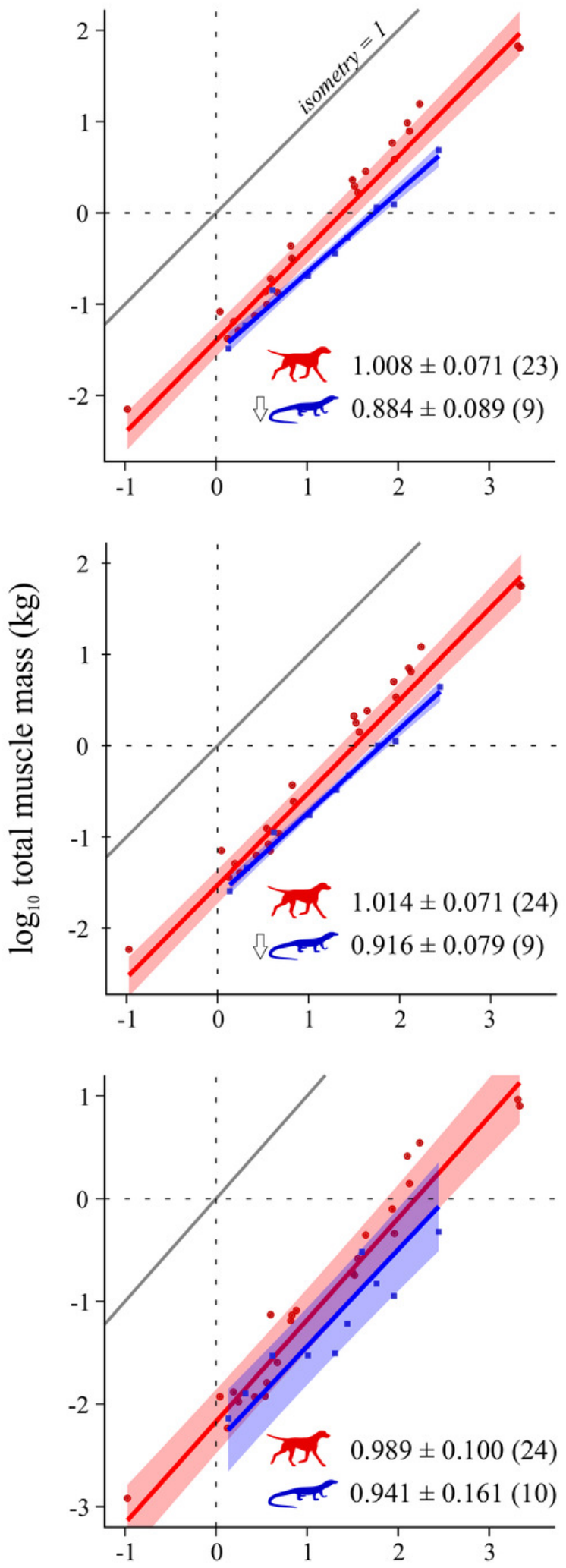

hindlimb
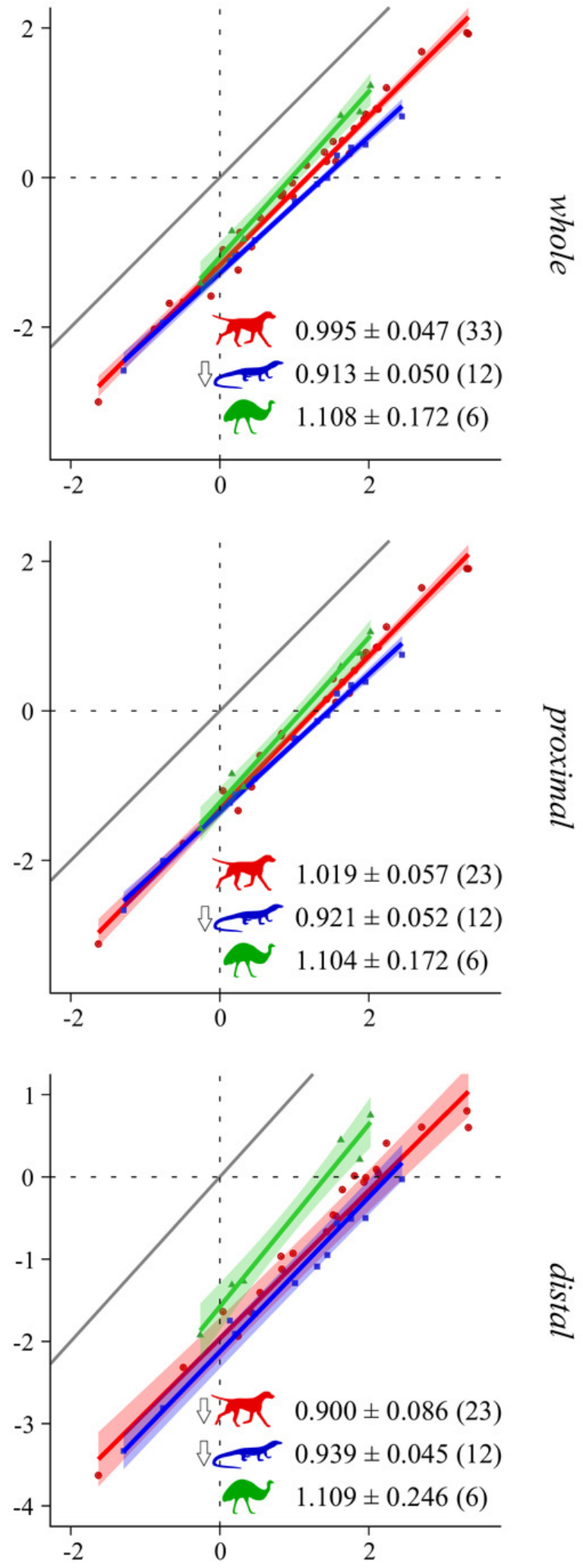

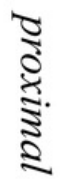<smiles>[C]1[C]=C1</smiles>

$\log _{10}$ body mass $(\mathrm{kg})$ 
Figure 3

Mean size-normalized isometric strength v. body mass across extant terrestrial amniotes.

Conventions as in Fig. 2. See Figs S2 and S3 for the results of median and total sizenormalized isometric strength versus body mass. 


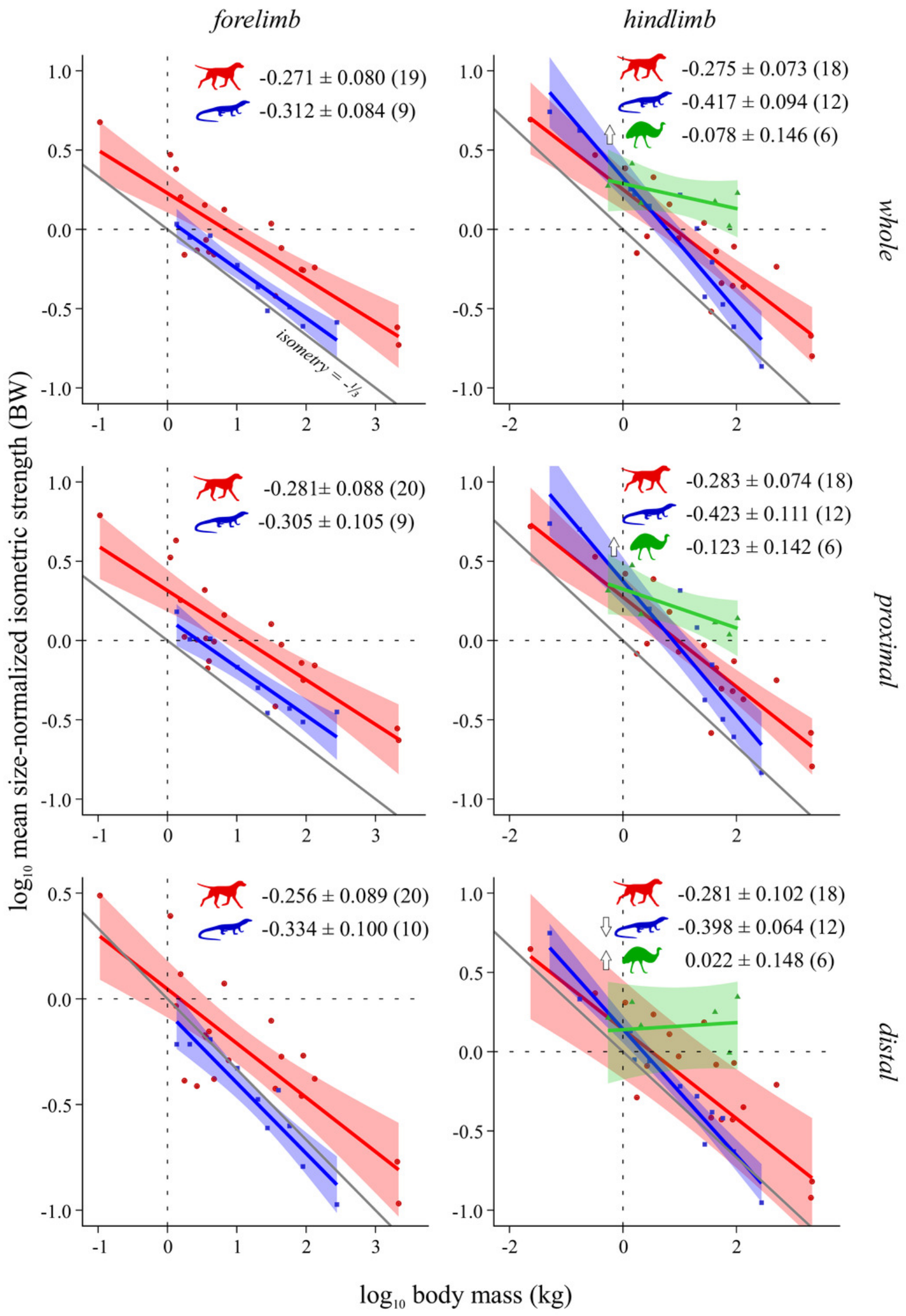


Figure 4

Characteristic fibre length $\mathrm{v}$. body mass across extant terrestrial amniotes.

Conventions as in Fig. 2. 
forelimb
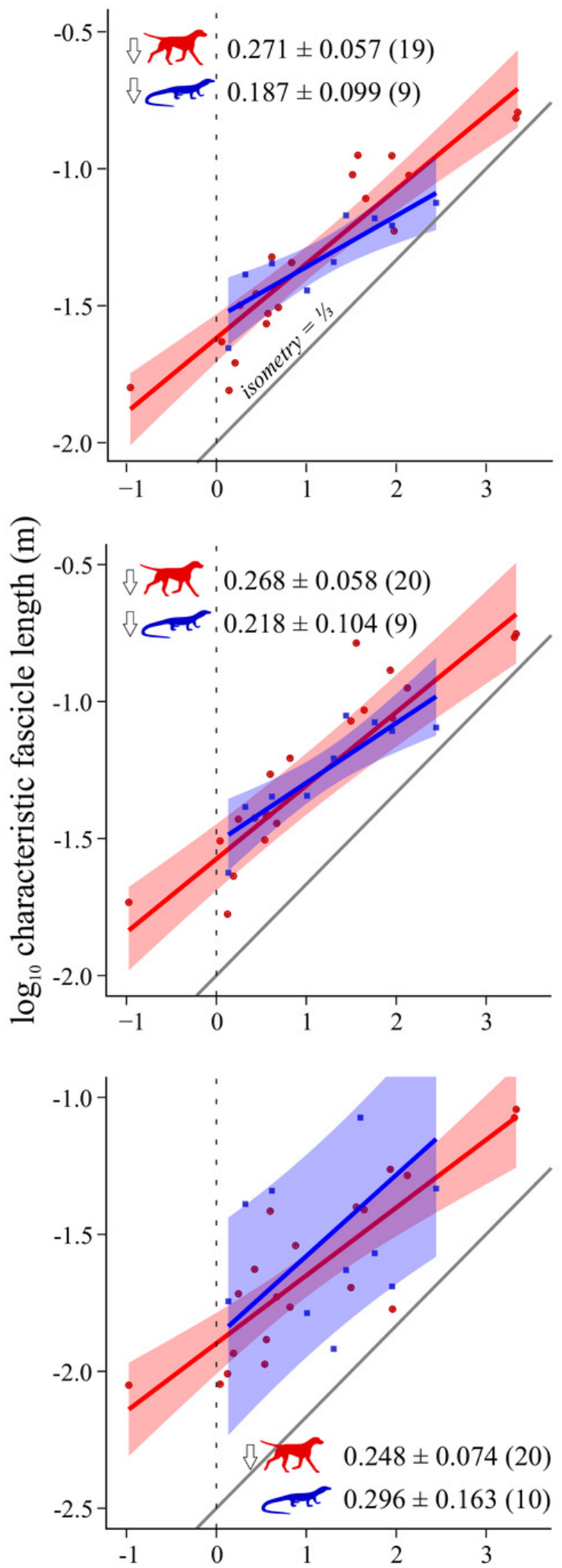

hindlimb
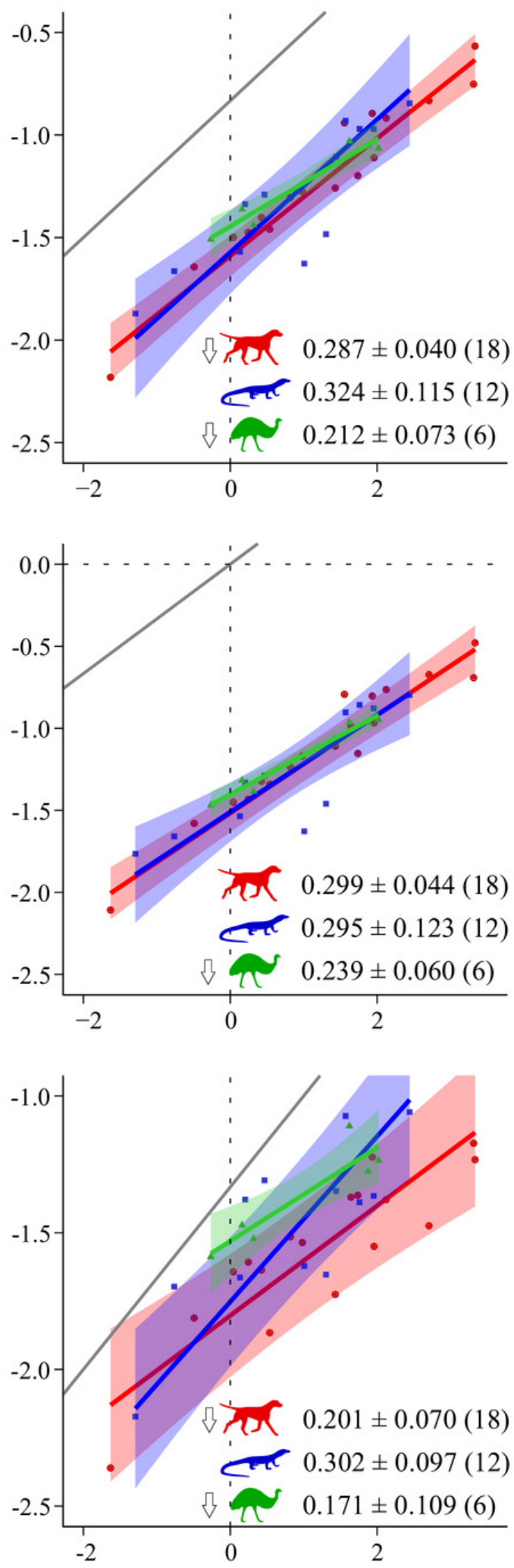

$\frac{\sqrt{2}}{\frac{1}{2}}$

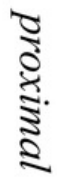

$\frac{2}{2}$

$\log _{10}$ body mass $(\mathrm{kg})$ 
Figure 5

Total physiological cross-sectional area v. total muscle mass across extant terrestrial amniotes.

Conventions as in Fig. 2. 
forelimb
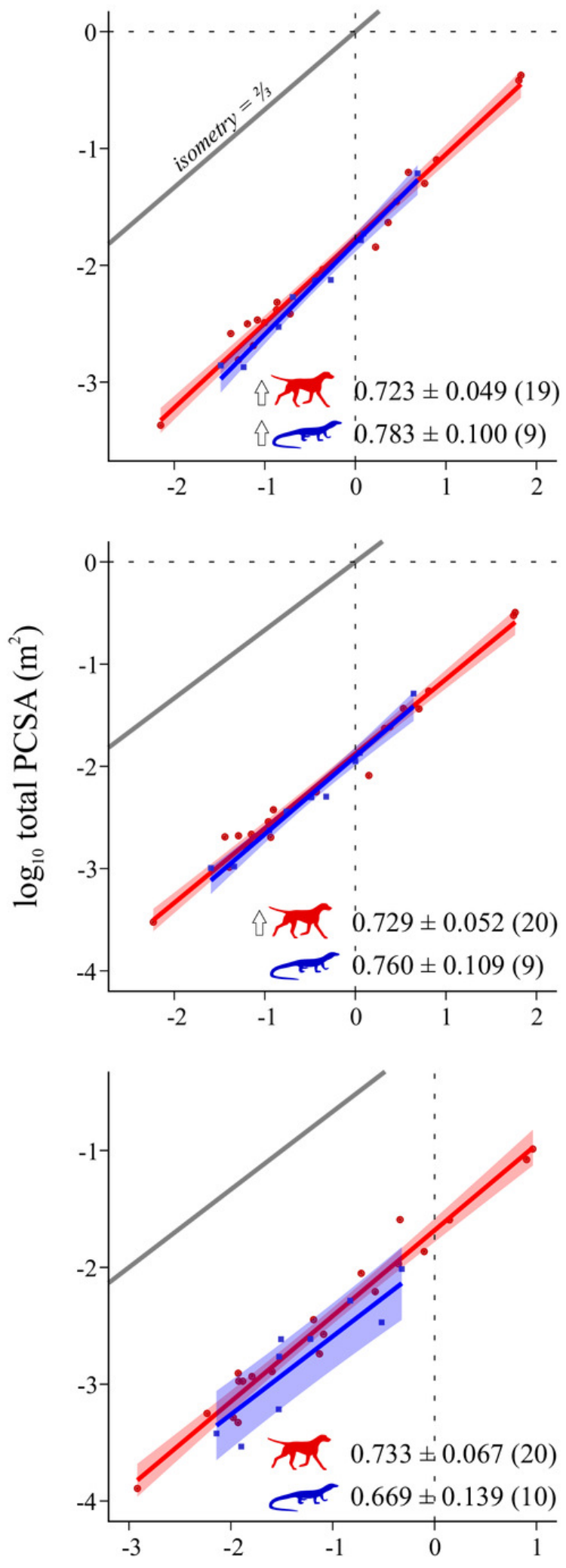

hindlimb
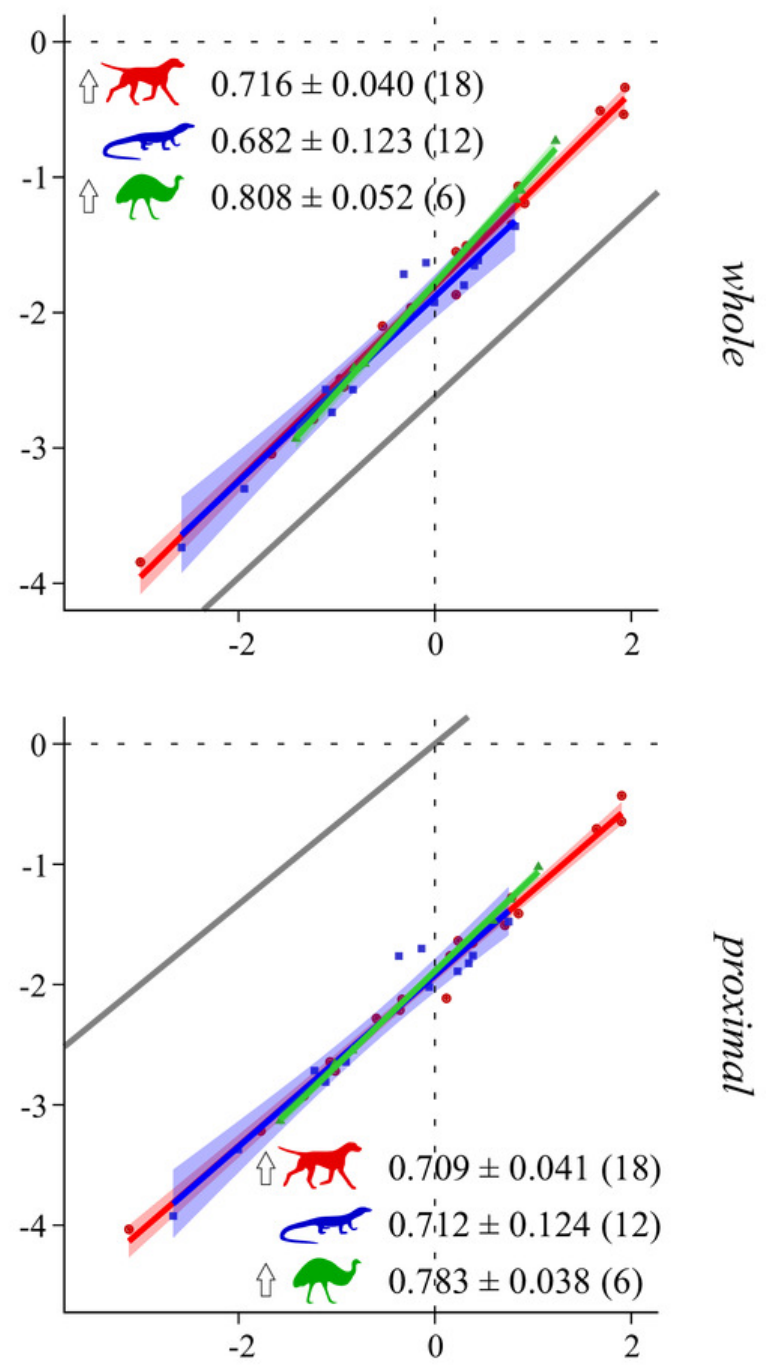

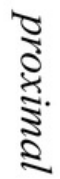

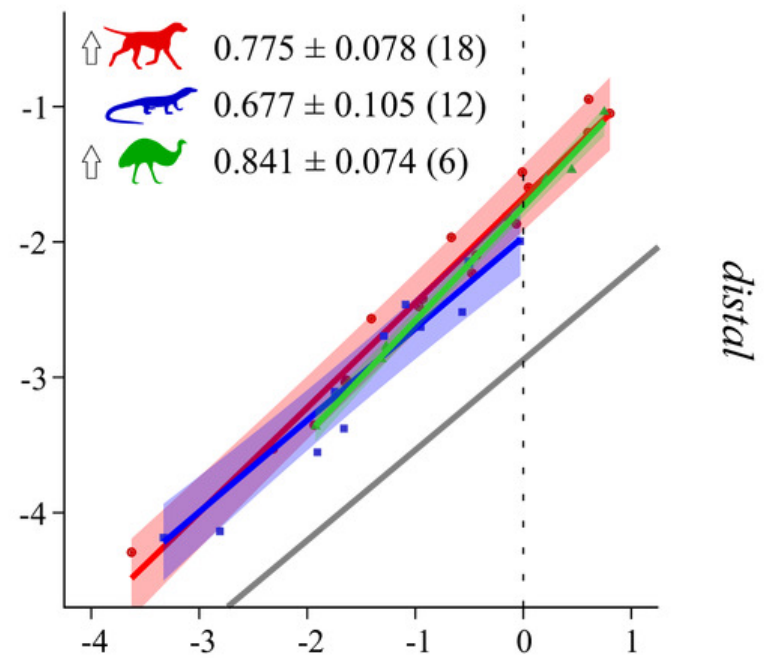

$\log _{10}$ total muscle mass $(\mathrm{kg})$ 


\section{Table $\mathbf{1}$ (on next page)}

Summary of data sources used in the study.

List of species with architectural data for individual muscles, the major group they belong to (for the purposes of the statistical analyses conducted here), body mass, whether a given species contributed to the forelimb ('fore') or hindlimb ('hind') datasets, and studies in which the data were originally published. Note that birds and humans were analysed together in this study as 'bipeds'. See Table S1 for additional species and studies that contributed data on muscle mass only. 
1 Table 1. Summary of data sources used in the study. List of species with architectural data

2 for individual muscles, the major group they belong to (for the purposes of the statistical

3 analyses conducted here), body mass, whether a given species contributed to the forelimb

4 ('fore') or hindlimb ('hind') datasets, and studies in which the data were originally published.

5 Note that birds and humans were analysed together in this study as 'bipeds'. See Table S1 for

6 additional species and studies that contributed data on muscle mass only.

\begin{tabular}{|c|c|c|c|c|}
\hline Species & Group & Body mass (kg) & Data & Study \\
\hline Osteolaemus tetraspis & reptile & 10.2 & fore, hind & Allen et al. (2015) \\
\hline Crocodylus johnstoni & reptile & 20.19 & fore, hind & Allen et al. (2015) \\
\hline Crocodylus moreletii & reptile & 27.7 & fore, hind & Allen et al. (2015) \\
\hline Alligator mississippiensis & reptile & 57.7 & fore, hind & Allen et al. (2015) \\
\hline Melanosuchus niger & reptile & 90 & fore, hind & Allen et al. (2015) \\
\hline Crocodylus niloticus & reptile & 278 & fore, hind & Allen et al. (2015) \\
\hline Varanus tristis & reptile & 0.173 & hind & Dick \& Clemente (2016) \\
\hline Varanus varius & reptile & 2.936 & hind & Dick \& Clemente (2016) \\
\hline Varanus panoptes & reptile & $4.15 / 1.6$ & fore, hind & Dick \& Clemente (2016), Cieri et al. (2020) \\
\hline Varanus komodoensis & reptile & $40 / 37.3$ & fore, hind & Dick \& Clemente (2016) \\
\hline Varanus spenceri & reptile & 2.098 & fore & Cieri et al. (2020) \\
\hline Salvator merianae & reptile & 1.359 & fore, hind & This study \\
\hline Basiliscus vittatus & reptile & 0.0511 & hind & Bergmann \& Hare-Drubka (2015) \\
\hline Eudromia elegans & biped & 0.545 & hind & Bishop et al. (2021b) \\
\hline Numida meleagris & biped & 1.45 & hind & Cox et al. (2019) \\
\hline Gallus gallus (junglefowl) & biped & 2.079 & hind & Paxton et al. (2010) \\
\hline Dromaius novaehollandiae & biped & 42 & hind & Lamas et al. (2014) \\
\hline Struthio camelus & biped & 105 & hind & Smith et al. (2006) \\
\hline Martes martes & mammal & 1.55 & fore & Böhmer et al. (2018) \\
\hline Taxidea taxus & mammal & 7.6 & fore & Moore et al. (2012) \\
\hline Canis familiaris & mammal & $31.4 / 27$ & fore, hind & Williams et al. (2008), Ellis et al. (2018) \\
\hline Felis nigripes & mammal & 1.1 & fore, hind & Cuff et al. (2016a),Cuff et al. (2016b) \\
\hline Felis silvestris & mammal & 2.66 & fore, hind & Cuff et al. (2016a), Cuff et al. (2016b) \\
\hline Leopardus pardalis & mammal & 9.6 & hind & Cuff et al. (2016b) \\
\hline Caracal caracal & mammal & 6.6 & fore, hind & Cuff et al. (2016a),Cuff et al. (2016b) \\
\hline Panthera uncia & mammal & 36 & fore, hind & Cuff et al. (2016a),Cuff et al. (2016b) \\
\hline Panthera onca & mammal & 44 & fore, hind & Cuff et al. (2016a),Cuff et al. (2016b) \\
\hline Panthera tigris & mammal & 86 & fore, hind & Cuff et al. (2016a), Cuff et al. (2016b) \\
\hline Panthera leo & mammal & 133 & fore, hind & Cuff et al. (2016a),Cuff et al. (2016b) \\
\hline Mus musculus & mammal & 0.02345 & hind & Charles et al. (2016) \\
\hline Rattus norvegicus & mammal & 0.323 & hind & Eng et al. (2008) \\
\hline Marmota monax & mammal & 4.7 & fore & Rupert et al. (2015) \\
\hline Equus caballus & mammal & 510 & hind & Payne et al. (2005) \\
\hline Ceratotherium simum & mammal & 2160 & fore, hind & Etienne et al. (2021) \\
\hline Rhinoceros unicornis & mammal & 2065 & fore, hind & Etienne et al. (2021) \\
\hline Lepus europeus & mammal & 3.454 & fore, hind & Williams et al. (2007a),Williams et al. (2007b) \\
\hline Scalopus aquaticus & mammal & 0.1062 & fore & Rose et al. (2013) \\
\hline Rangifer tarandus & mammal & 91 & fore, hind & Wareing et al. (2011) \\
\hline Homo sapiens & biped & 75.337 & hind & Rajagopal et al. (2016) \\
\hline Pan troglodytes & mammal & 54.7 & hind & Carlson (2006) \\
\hline Bradypus variegatus & mammal & 3.97 & fore & Olson et al. (2018) \\
\hline Dasypus novemcinctus & mammal & 3.6 & fore & Olson et al. (2016) \\
\hline Isoodon fusciventer & mammal & 1.329 & fore & Martin et al. (2019) \\
\hline Didelphis virginiana & mammal & 1.759 & fore, hind & This study \\
\hline Tachyglossus aculeatus & mammal & 3.79 & fore & Regnault et al. (2020) \\
\hline
\end{tabular}
7 


\section{Table 2 (on next page)}

Results of comparisons between each major group via pANCOVA, for the analysis of $\Sigma m_{\text {muscle }}$ v. $m_{\text {body }}$.

Each pairwise comparison was tested for differences in slope (S), intercept (I) and slope and intercept $(S+I)$. Results for analyses without controlling for phylogeny are also presented (ANCOVA, $\dagger$ ); significant results are in boldface; $d f=$ degrees of freedom. 
1 Table 2. Results of comparisons between each major group via pANCOVA, for the analysis of $\Sigma \boldsymbol{m}_{\text {muscle }} \mathbf{v}$. $\boldsymbol{m}_{\text {body }}$. Each pairwise comparison was tested for differences in slope (S), intercept (I) and slope and intercept (S + I). Results for analyses without controlling for phylogeny are also presented (ANCOVA, $\dagger$ ); significant results are in boldface; $d f=$ degrees of freedom. 4

\begin{tabular}{|c|c|c|c|c|c|c|c|c|c|c|c|c|c|c|c|c|c|}
\hline \multirow{2}{*}{\multicolumn{2}{|c|}{ anatomical region }} & \multirow{2}{*}{ test } & \multicolumn{5}{|c|}{ mammals v. reptiles } & \multicolumn{5}{|c|}{ mammals v. bipeds } & \multicolumn{5}{|c|}{ reptiles v. bipeds } \\
\hline & & & $d f$ & $F$ & $P$ & $F \dagger$ & $P \dagger$ & $d f$ & $F$ & $P$ & $F \dot{\dagger}$ & $P \dagger$ & $d f$ & $F$ & $P$ & $F \dagger$ & $P \dagger$ \\
\hline \multirow{9}{*}{ forelimb } & \multirow{3}{*}{ whole } & $S$ & 2,29 & 1.442 & 0.24 & 32.465 & $<0.001$ & & & & & & & & & & \\
\hline & & I & 2,29 & 1.639 & 0.211 & 33.14 & $<0.001$ & & & & & & & & & & \\
\hline & & $\mathrm{S}+\mathrm{I}$ & 2,28 & 1.08 & 0.353 & 19.112 & $<0.001$ & & & & & & & & & & \\
\hline & \multirow{3}{*}{ proximal } & $\mathrm{S}$ & 2,30 & 1.008 & 0.324 & 25.295 & $<0.001$ & & & & & & & & & & \\
\hline & & I & 2,30 & 1.114 & 0.3 & 27.243 & $<0.001$ & & & & & & & & & & \\
\hline & & $\mathrm{S}+\mathrm{I}$ & 2,29 & 0.73 & 0.491 & 14.967 & $<0.001$ & & & & & & & & & & \\
\hline & \multirow{3}{*}{ distal } & $S$ & 2,31 & 0.481 & 0.493 & 24.355 & $<0.001$ & & & & & & & & & & \\
\hline & & I & 2,31 & 0.509 & 0.481 & 17.047 & $<0.001$ & & & & & & & & & & \\
\hline & & $\mathrm{S}+\mathrm{I}$ & 2,30 & 0.377 & 0.689 & 11.945 & $<0.001$ & & & & & & & & & & \\
\hline \multirow{9}{*}{ hindlimb } & \multirow{3}{*}{ whole } & $\mathrm{S}$ & 2,42 & 1.673 & 0.203 & 14.715 & $<0.001$ & 2,36 & 5.181 & 0.029 & 11.763 & 0.002 & 2,15 & 21.811 & $<0.001$ & 41.475 & $<0.001$ \\
\hline & & I & 2,42 & 0.259 & 0.613 & 13.985 & 0.001 & 2,36 & 2.616 & 0.115 & 10.143 & 0.003 & 2,15 & 8.332 & 0.011 & 32.61 & $<0.001$ \\
\hline & & $\mathrm{S}+\mathrm{I}$ & 2,41 & 0.855 & 0.433 & 9.291 & 0.001 & 2,35 & 2.519 & 0.095 & 6.554 & 0.004 & 2,14 & 13.11 & 0.001 & 36.147 & $<0.001$ \\
\hline & \multirow{3}{*}{ proximal } & $\mathrm{S}$ & 2,32 & 4.268 & 0.047 & 16.724 & $<0.001$ & 2,26 & 3.688 & 0.066 & 6.595 & 0.016 & 2,15 & 7.192 & 0.017 & 10.129 & 0.006 \\
\hline & & I & 2,32 & 0.787 & 0.382 & 9.695 & 0.004 & 2,26 & 2.362 & 0.136 & 5.666 & 0.025 & 2,15 & 3.064 & 0.101 & 8.641 & 0.01 \\
\hline & & $\mathrm{S}+\mathrm{I}$ & 2,31 & 2.112 & 0.138 & 8.643 & 0.001 & 2,25 & 1.831 & 0.181 & 3.564 & 0.044 & 2,14 & 3.521 & 0.058 & 5.825 & 0.014 \\
\hline & \multirow{3}{*}{ distal } & S & 2,32 & 0.089 & 0.768 & 8.089 & 0.008 & 2,26 & 9.577 & 0.005 & 26.166 & $<0.001$ & 2,15 & 10.088 & 0.006 & 15.239 & 0.001 \\
\hline & & I & 2,32 & 0.199 & 0.659 & 15.57 & $<0.001$ & 2,26 & 5.05 & 0.033 & 26.659 & $<0.001$ & 2,15 & 6.737 & 0.02 & 31.079 & $<0.001$ \\
\hline & & $S+I$ & 2,31 & 0.166 & 0.848 & 7.819 & 0.002 & 2,25 & 4.64 & 0.019 & 17.517 & $<0.001$ & 2,14 & 6.955 & 0.008 & 17.242 & $<0.001$ \\
\hline
\end{tabular}




\section{Table 3(on next page)}

Results of comparisons between each major group via pANCOVA and ANCOVA, for the analysis of mean $F_{\max } * v$. $m_{\text {body }}$.

Conventions as per Table 2. 


\begin{tabular}{|c|c|c|c|c|c|c|c|c|c|c|c|c|c|c|c|c|c|}
\hline \multirow{2}{*}{\multicolumn{2}{|c|}{ anatomical region }} & \multirow{2}{*}{ test } & \multicolumn{6}{|c|}{ mammals v. reptiles } & \multicolumn{5}{|c|}{ mammals v. bipeds } & \multicolumn{4}{|c|}{ reptiles v. bipeds } \\
\hline & & & $d f$ & $F$ & $P$ & $F \dot{P}$ & $P \dagger$ & $d f$ & $F$ & $P$ & $F \dagger$ & $P \dagger$ & $d f$ & $F$ & $P$ & $F \dagger$ & $P_{\dagger}^{\prime}$ \\
\hline \multirow{9}{*}{ forelimb } & \multirow{3}{*}{ whole } & $\mathrm{S}$ & 2,25 & 1.212 & 0.282 & 9.013 & 0.006 & & & & & & & & & & \\
\hline & & I & 2,25 & 2.436 & 0.131 & 11.512 & 0.002 & & & & & & & & & & \\
\hline & & $\mathrm{S}+\mathrm{I}$ & 2,24 & 1.187 & 0.322 & 5.725 & 0.009 & & & & & & & & & & \\
\hline & \multirow{3}{*}{ proximal } & $\mathrm{S}$ & 2,26 & 0.673 & 0.419 & 6.247 & 0.019 & & & & & & & & & & \\
\hline & & I & 2,26 & 1.831 & 0.188 & 8.6 & 0.007 & & & & & & & & & & \\
\hline & & $\mathrm{S}+\mathrm{I}$ & 2,25 & 0.881 & 0.427 & 4.183 & 0.027 & & & & & & & & & & \\
\hline & \multirow{3}{*}{ distal } & $\mathrm{S}$ & 2,27 & 1.604 & 0.216 & 9.006 & 0.006 & & & & & & & & & & \\
\hline & & I & 2,27 & 1.554 & 0.223 & 8.939 & 0.006 & & & & & & & & & & \\
\hline & & $\mathrm{S}+\mathrm{I}$ & 2,26 & 0.942 & 0.403 & 4.824 & 0.017 & & & & & & & & & & \\
\hline \multirow{9}{*}{ hindlimb } & \multirow{3}{*}{ whole } & $\mathrm{S}$ & 2,27 & 6.699 & 0.015 & 6.699 & 0.015 & 2,21 & 14.358 & 0.001 & 14.358 & 0.001 & 2,15 & 35.374 & $<0.001$ & 35.374 & $<0.001$ \\
\hline & & I & 2,27 & 0.886 & 0.355 & 0.886 & 0.355 & 2,21 & 6.785 & 0.017 & 6.785 & 0.017 & 2,15 & 6.387 & 0.023 & 6.387 & 0.023 \\
\hline & & $\mathrm{S}+\mathrm{I}$ & 2,26 & 3.674 & 0.039 & 3.674 & 0.039 & 2,20 & 6.919 & 0.005 & 6.919 & 0.005 & 2,14 & 16.722 & $<0.001$ & 16.722 & $<0.001$ \\
\hline & \multirow{3}{*}{ proximal } & $\mathrm{S}$ & 2,27 & 4.379 & 0.046 & 4.379 & 0.046 & 2,21 & 10.455 & 0.004 & 10.455 & 0.004 & 2,15 & 19.785 & 0.001 & 19.785 & 0.001 \\
\hline & & I & 2,27 & 0.238 & 0.629 & 0.238 & 0.629 & 2,21 & 5.882 & 0.024 & 5.882 & 0.024 & 2,15 & 4.273 & 0.056 & 4.273 & 0.056 \\
\hline & & $\mathrm{S}+\mathrm{I}$ & 2,26 & 2.818 & 0.078 & 2.818 & 0.078 & 2,20 & 5.117 & 0.016 & 5.117 & 0.016 & 2,14 & 9.453 & 0.003 & 9.453 & 0.003 \\
\hline & \multirow{3}{*}{ distal } & $\mathrm{S}$ & 2,27 & 2.232 & 0.147 & 12.439 & 0.002 & 2,21 & 11.022 & 0.003 & 14.949 & 0.001 & 2,15 & 49.529 & $<0.001$ & 92.834 & $<0.001$ \\
\hline & & I & 2,27 & 0.096 & 0.76 & 7.245 & 0.012 & 2,21 & 3.055 & 0.095 & 6.169 & 0.022 & 2,15 & 3.88 & 0.068 & 16.093 & 0.001 \\
\hline & & $\mathrm{S}+\mathrm{I}$ & 2,26 & 1.075 & 0.356 & 6.432 & 0.005 & 2,20 & 5.402 & 0.013 & 7.131 & 0.005 & 2,14 & 23.132 & $<0.001$ & 47.383 & $<0.001$ \\
\hline
\end{tabular}

3 


\section{Table 4 (on next page)}

Results of comparisons between each major group via PANCOVA and ANCOVA, for the analysis of $L^{*} v$. $m_{\text {body }}$.

Conventions as per Table 2. 
1 Table 4. Results of comparisons between each major group via pANCOVA and ANCOVA, for the analysis of $L$ * $v$. $m_{\text {body }}$.

2 Conventions as per Table 2 .

\begin{tabular}{|c|c|c|c|c|c|c|c|c|c|c|c|c|c|c|c|c|c|}
\hline \multirow{2}{*}{\multicolumn{2}{|c|}{ anatomical region }} & \multirow{2}{*}{ test } & \multicolumn{5}{|c|}{ mammals v. reptiles } & \multicolumn{5}{|c|}{ mammals v. bipeds } & \multicolumn{5}{|c|}{ reptiles v. bipeds } \\
\hline & & & $d f$ & $F$ & $P$ & $F \dagger$ & $P_{\dagger}^{\dagger}$ & $d f$ & $F$ & $P$ & $F \dagger$ & $P \dagger$ & $d f$ & $F$ & $P$ & $F \dot{F}$ & $P_{\dagger}$ \\
\hline \multirow{9}{*}{ forelimb } & \multirow{3}{*}{ whole } & $\mathrm{S}$ & 2,25 & 1.537 & 0.227 & 1.537 & 0.227 & & & & & & & & & & \\
\hline & & I & 2,25 & 0.271 & 0.607 & 0.271 & 0.607 & & & & & & & & & & \\
\hline & & $\mathrm{S}+\mathrm{I}$ & 2,24 & 1.151 & 0.333 & 1.151 & 0.333 & & & & & & & & & & \\
\hline & \multirow{3}{*}{ proximal } & $\mathrm{S}$ & 2,26 & 1.251 & 0.274 & 1.251 & 0.274 & & & & & & & & & & \\
\hline & & I & 2,26 & 0.58 & 0.453 & 0.58 & 0.453 & & & & & & & & & & \\
\hline & & $\mathrm{S}+\mathrm{I}$ & 2,25 & 0.642 & 0.535 & 0.642 & 0.535 & & & & & & & & & & \\
\hline & \multirow{3}{*}{ distal } & $\mathrm{S}$ & 2,27 & 0.055 & 0.816 & 0.263 & 0.613 & & & & & & & & & & \\
\hline & & I & 2,27 & 0.113 & 0.74 & 0.288 & 0.596 & & & & & & & & & & \\
\hline & & $\mathrm{S}+\mathrm{I}$ & 2,26 & 0.062 & 0.94 & 1.921 & 0.167 & & & & & & & & & & \\
\hline \multirow{9}{*}{ hindlimb } & \multirow{3}{*}{ whole } & $\mathrm{S}$ & 2,27 & 0.395 & 0.535 & 0.025 & 0.875 & 2,21 & 0.379 & 0.545 & 0.073 & 0.789 & 2,15 & 0.603 & 0.45 & 0.013 & 0.91 \\
\hline & & I & 2,27 & 0.181 & 0.674 & 0.157 & 0.695 & 2,21 & 0.651 & 0.429 & 1.437 & 0.244 & 2,15 & 0.114 & 0.741 & 0.243 & 0.629 \\
\hline & & $\mathrm{S}+\mathrm{I}$ & 2,26 & 0.211 & 0.811 & 0.223 & 0.802 & 2,20 & 1.658 & 0.216 & 2.24 & 0.133 & 2,14 & 0.84 & 0.452 & 0.313 & 0.736 \\
\hline & \multirow{3}{*}{ proximal } & S & 2,27 & 0.002 & 0.964 & 0.794 & 0.381 & 2,21 & 0.269 & 0.609 & 0.175 & 0.68 & 2,15 & 0.041 & 0.842 & 0.094 & 0.764 \\
\hline & & I & 2,27 & 0.008 & 0.929 & 0.367 & 0.55 & 2,21 & 0.273 & 0.607 & 0.372 & 0.548 & 2,15 & 0.346 & 0.565 & 0.636 & 0.438 \\
\hline & & $\mathrm{S}+\mathrm{I}$ & 2,26 & 0.008 & 0.992 & 0.383 & 0.685 & 2,20 & 0.84 & 0.446 & 0.966 & 0.398 & 2,14 & 0.44 & 0.653 & 0.354 & 0.708 \\
\hline & \multirow{3}{*}{ distal } & $\mathrm{S}$ & 2,27 & 3.644 & 0.067 & 2.953 & 0.097 & 2,21 & 0.64 & 0.433 & 3.941 & 0.06 & 2,15 & 1.323 & 0.268 & 0.14 & 0.713 \\
\hline & & I & 2,27 & 0.277 & 0.603 & 5.02 & 0.034 & 2,21 & 1.846 & 0.189 & 11.95 & 0.002 & 2,15 & 0.422 & 0.526 & 1.106 & 0.31 \\
\hline & & $\mathrm{S}+\mathrm{I}$ & 2,26 & 1.782 & 0.188 & 2.519 & 0.1 & 2,20 & 0.928 & 0.412 & 5.75 & 0.011 & 2,14 & 1.663 & 0.225 & 0.635 & 0.545 \\
\hline
\end{tabular}




\section{Table 5 (on next page)}

Results of comparisons between each major group via pANCOVA and ANCOVA, for the analysis of $\Sigma$ PCSA v. $\Sigma m_{\text {muscle }}$.

Conventions as per Table 2. 
1 Table 5. Results of comparisons between each major group via pANCOVA and ANCOVA, for the analysis of $\Sigma$ PCSA v.

$2 \quad \boldsymbol{\Sigma} \boldsymbol{m}_{\text {muscle }}$ Conventions as per Table 2.

\begin{tabular}{|c|c|c|c|c|c|c|c|c|c|c|c|c|c|c|c|c|c|}
\hline \multirow{2}{*}{\multicolumn{2}{|c|}{ anatomical region }} & \multirow{2}{*}{ test } & \multicolumn{5}{|c|}{ mammals v. reptiles } & \multicolumn{5}{|c|}{ mammals v. bipeds } & \multicolumn{5}{|c|}{ reptiles v. bipeds } \\
\hline & & & $d f$ & $\boldsymbol{F}$ & $P$ & $F \dagger$ & $P \dagger$ & $d f$ & $F$ & $P$ & $F \dagger$ & $P_{\dagger}^{\dagger}$ & $d f$ & $F$ & $P$ & $F \dagger$ & $P_{\dagger}^{\dagger}$ \\
\hline \multirow{9}{*}{ forelimb } & \multirow{3}{*}{ whole } & $\mathrm{S}$ & 2,25 & 2.729 & 0.111 & 2.729 & 0.111 & & & & & & & & & & \\
\hline & & I & 2,25 & 2.081 & 0.162 & 2.081 & 0.162 & & & & & & & & & & \\
\hline & & $\mathrm{S}+\mathrm{I}$ & 2,24 & 1.591 & 0.225 & 1.591 & 0.225 & & & & & & & & & & \\
\hline & \multirow{3}{*}{ proximal } & $\mathrm{S}$ & 2,26 & 0.937 & 0.342 & 0.937 & 0.342 & & & & & & & & & & \\
\hline & & I & 2,26 & 0.901 & 0.351 & 0.901 & 0.351 & & & & & & & & & & \\
\hline & & $\mathrm{S}+\mathrm{I}$ & 2,25 & 0.572 & 0.572 & 0.572 & 0.572 & & & & & & & & & & \\
\hline & \multirow{3}{*}{ distal } & $\mathrm{S}$ & 2,27 & 0.01 & 0.92 & 3.383 & 0.077 & & & & & & & & & & \\
\hline & & I & 2,27 & 0.689 & 0.414 & 3.327 & 0.079 & & & & & & & & & & \\
\hline & & $\mathrm{S}+\mathrm{I}$ & 2,26 & 0.434 & 0.653 & 1.726 & 0.198 & & & & & & & & & & \\
\hline \multirow{9}{*}{ hindlimb } & \multirow{3}{*}{ whole } & $\mathrm{S}$ & 2,27 & 0.075 & 0.787 & 0.075 & 0.787 & 2,21 & 5.272 & 0.032 & 5.272 & 0.032 & 2,15 & 1.215 & 0.288 & 1.215 & 0.288 \\
\hline & & I & 2,27 & 1.265 & 0.271 & 1.265 & 0.271 & 2,21 & 0.225 & 0.64 & 0.225 & 0.64 & 2,15 & 0.642 & 0.435 & 0.642 & 0.435 \\
\hline & & $\mathrm{S}+\mathrm{I}$ & 2,26 & 0.611 & 0.551 & 0.611 & 0.551 & 2,20 & 2.676 & 0.093 & 2.676 & 0.093 & 2,14 & 1.124 & 0.353 & 1.124 & 0.353 \\
\hline & \multirow{3}{*}{ proximal } & $\mathrm{S}$ & 2,27 & 0.004 & 0.951 & 0.004 & 0.951 & 2,21 & 2.84 & 0.107 & 2.84 & 0.107 & 2,15 & 0.655 & 0.431 & 0.655 & 0.431 \\
\hline & & I & 2,27 & $1 \times 10^{-4}$ & 0.993 & $1 \times 10^{-4}$ & 0.993 & 2,21 & 0.313 & 0.582 & 0.313 & 0.582 & 2,15 & 0.028 & 0.869 & 0.028 & 0.869 \\
\hline & & $\mathrm{S}+\mathrm{I}$ & 2,26 & 0.002 & 0.998 & 0.002 & 0.998 & 2,20 & 1.743 & 0.201 & 1.743 & 0.201 & 2,14 & 0.392 & 0.683 & 0.392 & 0.683 \\
\hline & \multirow{3}{*}{ distal } & $\mathrm{S}$ & 2,27 & 1.466 & 0.237 & 3.118 & 0.089 & 2,21 & 1.027 & 0.323 & 2.911 & 0.103 & 2,15 & 4.137 & 0.06 & 0.146 & 0.708 \\
\hline & & I & 2,27 & 0.505 & 0.483 & 9.851 & 0.004 & 2,21 & 0.195 & 0.663 & 3.387 & 0.08 & 2,15 & 0.233 & 0.636 & 0.738 & 0.404 \\
\hline & & $\mathrm{S}+\mathrm{I}$ & 2,26 & 1.628 & 0.216 & 4.994 & 0.015 & 2,20 & 0.551 & 0.585 & 2.141 & 0.144 & 2,14 & 3.242 & 0.07 & 1.062 & 0.372 \\
\hline
\end{tabular}




\section{Table 6(on next page)}

Pan-amniote regression ( $p G L S$ ) coefficients for each comparison.

These are reported for data on a log10 scale; also reported is the mean percent prediction error (\%PE), expressed in original (antilog) terms. Results for hindlimb analyses where the caudofemoralis longus was excluded from the reptile dataset are reported in Table S10. 
Table 6. Pan-amniote regression (pGLS) coefficients for each comparison. These are reported for data on a log 10 scale; also reported is the mean percent prediction error $(\% \mathrm{PE})$, expressed in original (antilog) terms. Results for

\begin{tabular}{|c|c|c|c|c|c|c|c|c|c|c|c|}
\hline \multirow[b]{2}{*}{ comparison } & \multirow{2}{*}{$\begin{array}{l}\text { slope under } \\
\text { isometry }\end{array}$} & \multirow[b]{2}{*}{$\operatorname{limb}$} & \multicolumn{3}{|c|}{ whole limb } & \multicolumn{3}{|c|}{ proximal } & \multicolumn{3}{|c|}{ distal } \\
\hline & & & slope & intercept & $\begin{array}{l}\text { mean } \\
\% P E\end{array}$ & slope & intercept & $\begin{array}{l}\text { mean } \\
\% \text { PE }\end{array}$ & slope & intercept & $\begin{array}{l}\text { mean } \\
\% \text { PE }\end{array}$ \\
\hline \multirow{2}{*}{$\Sigma m_{\text {muscle }}$ V. $m_{\text {body }}$} & \multirow{2}{*}{1.0} & fore & 1.0009 & -1.5459 & 64.8 & 1.0076 & -1.6512 & 66.52 & 0.9786 & -2.3141 & 83.01 \\
\hline & & hind & 0.9798 & -1.2764 & 40.77 & 0.9975 & -1.3619 & 32.84 & 0.9069 & -2.0589 & 55.32 \\
\hline \multirow{2}{*}{$\begin{array}{l}\text { mean } F_{\max } * \mathrm{v} . \\
m_{\text {body }}\end{array}$} & \multirow{2}{*}{-0.333} & fore & -0.2798 & 0.1213 & 43.11 & -0.2871 & 0.2092 & 46.12 & -0.276 & -0.0573 & 45.03 \\
\hline & & hind & -0.3177 & 0.2791 & 33.58 & -0.3266 & 0.3149 & 35.28 & -0.3046 & 0.1345 & 45.66 \\
\hline \multirow{2}{*}{$\begin{array}{l}\operatorname{median} F_{\max } * \mathrm{v} . \\
m_{\text {body }}\end{array}$} & \multirow{2}{*}{-0.333} & fore & -0.3045 & -0.0375 & 38.71 & -0.3174 & 0.0782 & 44.67 & -0.282 & -0.1878 & 46.31 \\
\hline & & hind & -0.2916 & 0.0154 & 40.38 & -0.2757 & 0.0449 & 44.48 & -0.3027 & -0.0581 & 42.02 \\
\hline \multirow{2}{*}{$\Sigma F_{\max } * \mathrm{v} . m_{\text {body }}$} & \multirow{2}{*}{-0.333} & fore & -0.2896 & 1.5756 & 48.65 & -0.2755 & 1.4068 & 48.2 & -0.3146 & 1.0427 & 66.34 \\
\hline & & hind & -0.307 & 1.7461 & 40.79 & -0.2988 & 1.6095 & 36.11 & -0.3314 & 1.1473 & 67.41 \\
\hline \multirow{2}{*}{$L^{*}$ v. $m_{\text {body }}$} & \multirow{2}{*}{0.333} & fore & 0.2559 & -1.6124 & 23.32 & 0.2603 & -1.5407 & 21.54 & 0.2607 & -1.8702 & 41.57 \\
\hline & & hind & 0.2943 & -1.5699 & 21.46 & 0.3016 & -1.5117 & 21.91 & 0.2296 & -1.7568 & 35.41 \\
\hline \multirow{2}{*}{$\begin{array}{l}\Sigma \text { PCSA v. } \\
\Sigma m_{\text {muscle }}\end{array}$} & \multirow{2}{*}{0.666} & fore & 0.7344 & -1.7894 & 18.82 & 0.735 & -1.8876 & 18.46 & 0.7215 & -1.7914 & 38.93 \\
\hline & & hind & 0.7141 & -1.8341 & 26.48 & 0.7095 & -1.9253 & 26.2 & 0.746 & -1.795 & 36.97 \\
\hline
\end{tabular}


